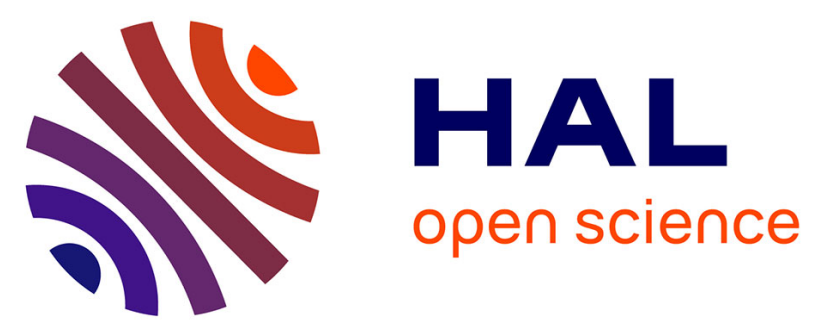

\title{
Combining density functional theory and Monte Carlo neutron transport calculations to study the phonon density of states of UO2 up to $1675 \mathrm{~K}$ by inelastic neutron scattering
}

Gilles Noguere, J.P. Scotta, Shuqi Xu, A. Filhol, Jacques Ollivier, Emmanuel Farhi, Yoann Calzavarra, Stéphane Rols, Bjorn Fak, Jean-Marc Zanotti, et al.

\section{To cite this version:}

Gilles Noguere, J.P. Scotta, Shuqi Xu, A. Filhol, Jacques Ollivier, et al.. Combining density functional theory and Monte Carlo neutron transport calculations to study the phonon density of states of UO2 up to $1675 \mathrm{~K}$ by inelastic neutron scattering. Physical Review B, 2020, 102, 10.1103/physrevb.102.134312 . hal-02997948

\section{HAL Id: hal-02997948 \\ https://hal.science/hal-02997948}

Submitted on 10 Nov 2020

HAL is a multi-disciplinary open access archive for the deposit and dissemination of scientific research documents, whether they are published or not. The documents may come from teaching and research institutions in France or abroad, or from public or private research centers.
L'archive ouverte pluridisciplinaire HAL, est destinée au dépôt et à la diffusion de documents scientifiques de niveau recherche, publiés ou non, émanant des établissements d'enseignement et de recherche français ou étrangers, des laboratoires publics ou privés. 


\title{
Combining density functional theory and Monte Carlo neutron transport calculations to study the phonon density of states of $\mathrm{UO}_{2}$ up to $1675 \mathrm{~K}$ by inelastic neutron scattering
}

\author{
G. Noguere, J. P. Scotta, and S. Xu \\ CEA, DES, IRESNE, DER, Cadarache, F-13108 Saint Paul Les Durance, France \\ A. Filhol, J. Ollivier, E. Farhi, Y. Calzavarra, S. Rols, and B. Fak \\ Institut Laue-Langevin, F-38042 Grenoble, France \\ J.-M. Zanotti \\ Laboratoire Léon Brillouin (CEA-CNRS), Université Paris-Saclay, CEA Saclay, 91191 Gif-sur-Yvette Cedex, France \\ Q. Berrod \\ CNRS-CEA-Université Grenoble Alpes, SyMMES, 38000 Grenoble, France
}

(Received 5 June 2020; revised 26 August 2020; accepted 29 September 2020; published 30 October 2020)

\begin{abstract}
Significant advances in the use of atomistic simulation techniques, such as $a b$ initio density functional theory and the molecular dynamics method, made it possible to predictively calculate properties of materials. In parallel, low-energy neutron scattering instruments and data analysis tools available in different institutes become mature for providing high-quality data for experimental validation purposes. Despite such experimental and theoretical improvements, the accurate modeling of experimental neutron-weighted multiphonon spectra for $\mathrm{UO}_{2}$ over a broad temperature range still remains an issue. Combining prior phonon density of states (PDOS) from density functional theory and Monte Carlo inelastic neutron scattering calculations in a Bayesian fitting procedure is a valuable alternative approach to assess the partial PDOS of uranium and oxygen in $\mathrm{UO}_{2}$ from 294 to $1675 \mathrm{~K}$ for improving the comparison to experiment and exploring the first-principles calculation hypothesis.
\end{abstract}

DOI: 10.1103/PhysRevB.102.134312

\section{INTRODUCTION}

The complex insulating nature of uranium dioxide $\left(\mathrm{UO}_{2}\right)$ has been the subject of many experimental and theoretical studies since the pioneer works reported in the 1960s. At that time, major results about the vibration modes of $\mathrm{UO}_{2}$ were published in a series of symposia on neutron scattering and neutron thermalization organized by the International Atomic Energy Agency, between 1960 and 1977 [1,2]. Over the past 20 years, the first-principles density functional theory became an invaluable resource for supplying material properties to simulate the neutron slowing down in nuclear fuels.

The latest version of the United States evaluated nuclear data library, namely ENDF\B-VIII [3], benefited from the extensive efforts led by the Low Energy Interaction Physics group of the North Carolina State University to accommodate condense matter physics into the low-energy neutron-induced cross-section formalisms [4]. In the case of $\mathrm{UO}_{2}$, the VASP code [5] has been used to generate phonon density of states (PDOS) [6] for the ENDFVB library, replacing those established from the work of Dolling [7]. The corresponding PDOS for $T=0 \mathrm{~K}$ is shown in Fig. 1. The five normal modes of vibration of $\mathrm{UO}_{2}$ can be distinguished around $10 \mathrm{meV}, 20 \mathrm{meV}$, $30 \mathrm{meV}, 55 \mathrm{meV}$, and $70 \mathrm{meV}$. The two first peaks correspond to the translational and longitudinal acoustic phonon modes of the uranium atoms. The highest energy structures are dominated by the optical phonon modes of the oxygen atoms. For comparison, the PDOS calculated at Uppsala University for $T=294 \mathrm{~K}$ [8] is shown in the same figure. The principle of the calculations performed in the quasiharmonic approximation is reported in Refs. $[9,10]$ together with validation results. It consists of applying the finite-displacement method to a $\mathrm{UO}_{2}$ supercell through the PHONOPY code [11]. The interatomic forces are provided by the VASP code within the framework of the first-principles density functional theory. The comparison of the two ab initio PDOS highlights the phonon mode shift to lower energy transfer with increasing temperature. It also shows how the calculations performed at the Uppsala University are able to capture the splitting between the TO1 and LO1 optical phonon branches around 28 and $33 \mathrm{meV}$.

The results reported by Pang et al. $[12,13]$ represent the latest theoretical and experimental achievements of interest for our work. If first-principles PDOS simulations successfully reproduce inelastic neutron scattering data of $\mathrm{UO}_{2}$ measured at $294 \mathrm{~K}$ using the ARCS spectrometer of the Spallation Neutron Source (SNS), similar simulations still fail to reproduce high-temperature neutron-weighted phonon density of states $(T=1200 \mathrm{~K})$ which are characterized by a stronger anharmonic linewidth broadening [14]. These theoretical limitations make questionable the simulation of the neutron transport in nuclear fuel loaded in power reactors operating at hot full power. Indeed, the slowing down of neutrons in $\mathrm{UO}_{2}$ is affected by the vibrations of the uranium and oxygen atoms from which emerge neutron up-scattering effects that 


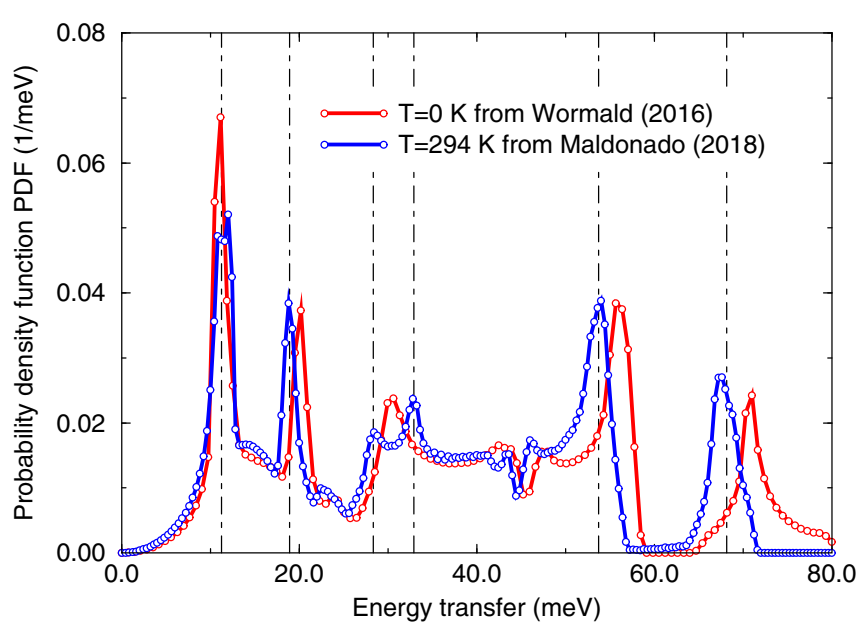

FIG. 1. Ab initio phonon density of states of $\mathrm{UO}_{2}$ at $T=0 \mathrm{~K}$ [6] and $T=294 \mathrm{~K}$ [8]. The dot-dashed lines locate the main vibration modes of $\mathrm{UO}_{2}$ calculated at $T=294 \mathrm{~K}$. The two first peaks at 10 and $20 \mathrm{meV}$ correspond to the translational (TA) and longitudinal (LA) acoustic phonon modes of the uranium atoms. The structures at around $30 \mathrm{meV}, 55 \mathrm{meV}$, and $70 \mathrm{meV}$ are dominated by the optical phonon modes (TO1, LO1, TO2, LO2) of the oxygen atoms.

modify the ${ }^{238} \mathrm{U}$ radiative capture reaction rate involving sizable changes on the neutron multiplication factor, Doppler reactivity coefficient, and ${ }^{239} \mathrm{Pu}$ inventory [15].

The present work aims to derive reliable temperaturedependent partial PDOS for oxygen and uranium in $\mathrm{UO}_{2}$ from inelastic neutron scattering data measured at the IN4 and IN6 time-of-flight spectrometers of the Institute Laue-Langevin (ILL) between 294 and $1675 \mathrm{~K}$. The originality of the work relies on the use of the Monte Carlo neutron transport code TRIPOLI4 [16] to simulate the experimental results by taking into account the multiple neutron scattering effects and the multiphonon contribution. The main prior input parameters are the partial phonon density of states for oxygen and uranium in $\mathrm{UO}_{2}$ provided by ab initio calculations. The obtained results are expected to stimulate advanced theoretical studies for improving comparison to experiment over a broad temperature range of interest for nuclear applications.

The main expressions involved in the neutron scattering formalism are reported in Sec. II. Section III briefly describes the measurements performed at ILL. The experimental neutron-weighted multiphonon spectra of $\mathrm{UO}_{2}$ as a function of the temperature are reported in Sec. IV. The interpretation of the data with the Monte Carlo neutron transport code TRIPOLI4 is presented in Sec. V. The obtained results are discussed in Sec. VI.

\section{NEUTRON SCATTERING FORMALISM}

In the low neutron energy range, typically below $5 \mathrm{eV}$, the slowing down of neutrons in $\mathrm{UO}_{2}$ depends on the energy and angular distribution of the scattering process. The mathematical expression of the corresponding double-differential neutron scattering cross section is well described in the literature. The main equations are summarized in Ref. [17]. They were obtained in the framework of the incoherent scattering approximation that allows one to neglect the interference terms in the case of polycrystalline materials and to express the double-differential neutron scattering cross section of each atom $X$ separately. If $T$ is the temperature of the target and $k_{B}$ is the Boltzmann constant, the temperature-dependent doubledifferential scattering cross section for $X\left({ }^{238} \mathrm{U}\right.$ or $\left.{ }^{16} \mathrm{O}\right)$ in $\mathrm{UO}_{2}$ can be written as [18]

$$
\frac{d^{2} \sigma_{n_{X}}^{T}}{d E^{\prime} d \theta}=\frac{\sigma_{n_{X}}^{0}}{2 k_{B} T} \sqrt{\frac{E^{\prime}}{E}} e^{-\beta / 2} S_{X}^{T}(\alpha, \beta),
$$

where $E$ is the neutron incident energy, $E^{\prime}$ stands for the energy of the scattered neutron, $\sigma_{n_{X}}^{0}$ is the bound neutron elastic scattering cross section at $T=0$, and $S_{X}^{T}(\alpha, \beta)$ is the symmetric form of the dynamic structure factor [19] which is defined as a function of the dimensionless variables $\alpha$ and $\beta$. The latter variables are related to the momentum transfer $q$ :

$$
\alpha=\frac{q^{2} \hbar^{2}}{2 M_{X} k_{B} T}=\frac{E^{\prime}+E-2 \mu \sqrt{E^{\prime} E}}{A_{X} k_{B} T},
$$

and energy transfer $\varepsilon=\hbar \omega$ :

$$
\beta=-\frac{\hbar \omega}{k_{B} T}=-\frac{E-E^{\prime}}{k_{B} T},
$$

where $\mu=\cos (\theta)$ is the cosine of the scattering angle in the laboratory system and $A_{X}$ is the ratio of the mass $M_{X}$ of the scattering atom $X$ to the neutron mass. An analytical form of $S_{X}^{T}(\alpha, \beta)$ was established for a cubic symmetry by using an isotropic harmonic potential which simplifies the complex averaging over all the possible orientations of the molecules. This model, known as the incoherent Gaussian approximation [20], led to the conventionally called phonon expansion:

$$
S_{X}^{T}(\alpha, \beta)=e^{-\alpha \lambda_{s}} \sum_{n=1}^{\infty} \frac{1}{n !}\left(\alpha \lambda_{s}\right)^{n} \mathcal{T}_{n}(\beta),
$$

where $n$ represents the phonon expansion order, $\lambda_{s}$ stands for the Debye-Waller coefficient, and $\mathcal{T}_{n}(\beta)$ has the generic form:

$$
\mathcal{T}_{n}(\beta)=\int_{-\infty}^{\infty} \mathcal{T}_{1}\left(\beta^{\prime}\right) \mathcal{T}_{n-1}\left(\beta-\beta^{\prime}\right) d \beta^{\prime} .
$$

The one-phonon term $\mathcal{T}_{1}(\beta)$ is given by

$$
\mathcal{T}_{1}(\beta)=\frac{\rho_{X}^{T}(\beta) e^{-\beta / 2}}{2 \lambda_{s} \beta \sinh (\beta / 2)} .
$$

This term explicitly depends on the partial phonon density of states $\rho_{X}^{T}(\beta)$. The aim of this study is to extract reliable values of $\rho_{X}^{T}(\beta)$ from the experimental quantity $\rho_{\exp }^{T}(\beta)$ which represents the neutron-weighted multiphonon spectrum of $\mathrm{UO}_{2}$ measured at ILL as a function of the temperature. A suitable expression of $\rho_{\exp }^{T}(\beta)$ can be obtained in the framework of the incoherent elastic one-phonon approximation. By introducing Eq. (6) in Eq. (4) for $n=1$ and by removing the contribution of the elastic peak, we obtain

$$
\rho_{\exp }^{T}(\beta) \simeq \lim _{\alpha \rightarrow 0} P_{\exp }^{T}(\alpha, \beta),
$$

with

$$
P_{\exp }^{T}(\alpha, \beta) \simeq \frac{2 \beta \sinh \beta / 2}{\alpha} S_{\exp }^{T}(\alpha, \beta),
$$

where $S_{\exp }^{T}$ represents the experimental symmetric form of the dynamic structure factor [Eq. (1)] without the contribution of 


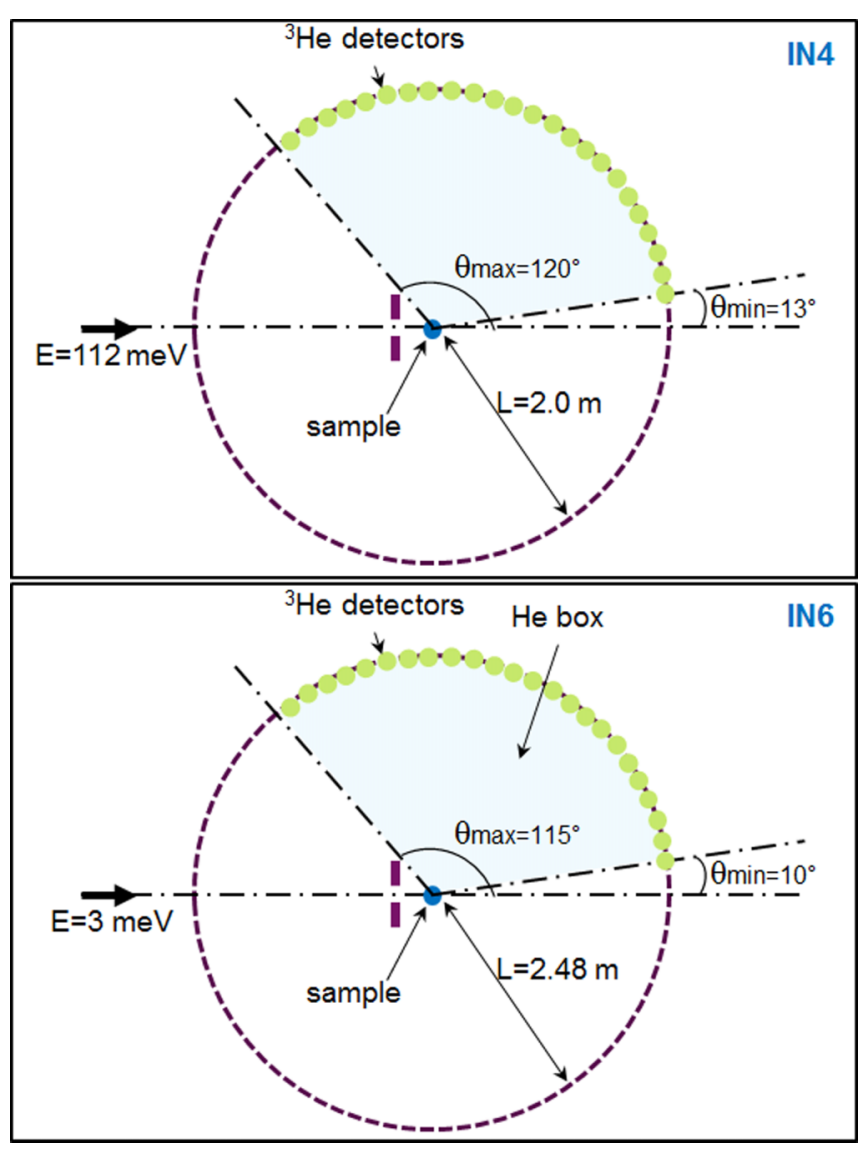

FIG. 2. Simplified top view of the IN4 and IN6 time-of-flight spectrometers of the Institute Laue-Langevin.

the quasielastic neutron scattering peak $S_{0}^{T}$. It is related to the experimental neutron yield $Y_{\exp }^{T}$ as follows:

$$
S_{\exp }^{T}(\alpha, \beta)=Y_{\exp }^{T}(\alpha, \beta) \sqrt{\frac{E}{E^{\prime}}} e^{\beta / 2}-S_{0}^{T}(\alpha, \beta) .
$$

\section{INELASTIC NEUTRON SCATTERING EXPERIMENTS}

Two experimental campaigns were undertaken in 2016 and 2019 to measure the experimental neutron yields $Y_{\exp }^{T}$ at 294, $300,350,420,600,900,1200$, and $1675 \mathrm{~K}$ on the IN6 and IN4 time-of-flight spectrometers of ILL for two incident neutron energies $E=3 \mathrm{meV}(\lambda=5.1 \AA)$ and $E=112 \mathrm{meV}(\lambda=$ $0.85 \AA)$, respectively. Experimental details and preliminary results were reported in Refs. [21,22].

From the point of view of the neutron transport physics, both spectrometers share the same basic design (Fig. 2). The neutron time-of-flight technique consists of measuring the time $t$ traveled by the neutrons from the sample until their detection at a given scattering angle $\theta$. If the energies are in $\mathrm{eV}$, the length in $\mathrm{m}$, and the time in $\mu \mathrm{s}$, the nonrelativistic time-energy relation $E^{\prime}=(72.298 L / t)^{2}$ is used to obtain the experimental neutron yield $Y_{\exp }^{T}\left(\theta, E^{\prime}\right)$ as a function of the outgoing neutron energies $E^{\prime}$. The flight distance between the sample and the detection setup is equal to $L=2.00$ (1) $\mathrm{m}$ and $L=2.48(1) \mathrm{m}$, for IN4 and IN6, respectively. Each ${ }^{3} \mathrm{He}$ detector array covers scattering angles ranging from approximately $10^{\circ}$ to $120^{\circ}$. In both cases, a rectangular monoenergetic neutron beam was focused to the sample, which was mounted in a high-temperature furnace placed at the center of the spectrometer. The sample is a 4.09(1)-cm-long cylinder composed of a stack of four depleted UOX pellets of 8.26(1) $\mathrm{mm}$ diameter. The total weight of the sample is 23.642(1) g, with a mass-fraction composition in ${ }^{238} \mathrm{U},{ }^{236} \mathrm{U},{ }^{235} \mathrm{U},{ }^{234} \mathrm{U}$, and ${ }^{16} \mathrm{O}$ of $87.598(1) \%$, $0.005(1) \% 0.264(1) \% 0.002(1) \%$, and $12.131(1) \%$, respectively. For the first experimental campaign, the $\mathrm{UO}_{2}$ sample was sealed in a quartz tube under primary vacuum and encapsulated in a thin niobium sample-holder tube. For the second one, the $\mathrm{UO}_{2}$ sample was simply encapsulated in a thick niobium sample-holder tube. Empty "dummy" samples were prepared to measure the background contribution.

The experiments consisted of a sequence of $\mathrm{UO}_{2}$ and "dummy" sample measurements, including a short irradiation of a vanadium sample. As this material nearly behaves as a pure incoherent elastic scatterer, its elastic scattering peak is used as a reference for the purpose of calibration and detector efficiency correction. The data reduction steps were handled with the ILL in-house LAMP code, except for the background correction. Indeed, replacing depleted UOX pellets by void in the "dummy" sample cannot ensure an accurate background subtraction due to significant sample absorption and multiple neutron scatterings. The background was then defined as the sum of the "dummy" sample data times an effective transmission factor plus an additional contribution due to neutrons scattered by the sample-holder tube and furnace environment. The effective transmission factor and contributions of the sample environment have been estimated with the Monte Carlo neutron transport code TRIPOLI4 [16], using the models shown in Fig. 2 and the neutron scattering formalism described in Eqs. (1)-(6).

\section{EXPERIMENTAL NEUTRON-WEIGHTED MULTIPHONON SPECTRUM}

The experimental neutron-weighted multiphonon spectra $\rho_{\exp }^{T}$ of $\mathrm{UO}_{2}$ were deduced from the temperature-dependent neutron yields $Y_{\exp }^{T}$ measured on IN6 by using the expression (7). In view of obtaining $P_{\exp }^{T}(\alpha, \beta)$ at $\alpha=0$, we have smoothed out fluctuations due to nuclei dynamics by using a $\log$ - $\log$ extrapolation to zero of the cumulative distribution functions of $P_{\exp }^{T}(\alpha, \beta)$ over $\alpha$. The contribution of the quasielastic neutron scattering peak $S_{0}^{T}$ was removed from the data using a pseudovoigt function. In the present analysis, no attempt was made to iteratively correct the data from the multiphonon contribution and the multiple neutron scattering effects, as usually proposed in the literature [23]. These two effects have different physical origins which will be included in the theoretical calculations. The multiphonon correction is mainly a temperature effect which is part of the dynamic structure factor. It is taken into account with the phonon expansion given by Eq. (4). The contribution of the scattered neutrons depends on several experimental factors such as the sample size and its environment. Such a correction is handled through Monte Carlo simulations whose principles are presented in Sec. V.

Figure 3 shows the neutron-weighted multiphonon spectrum of $\mathrm{UO}_{2}$ and the background contributions measured on 

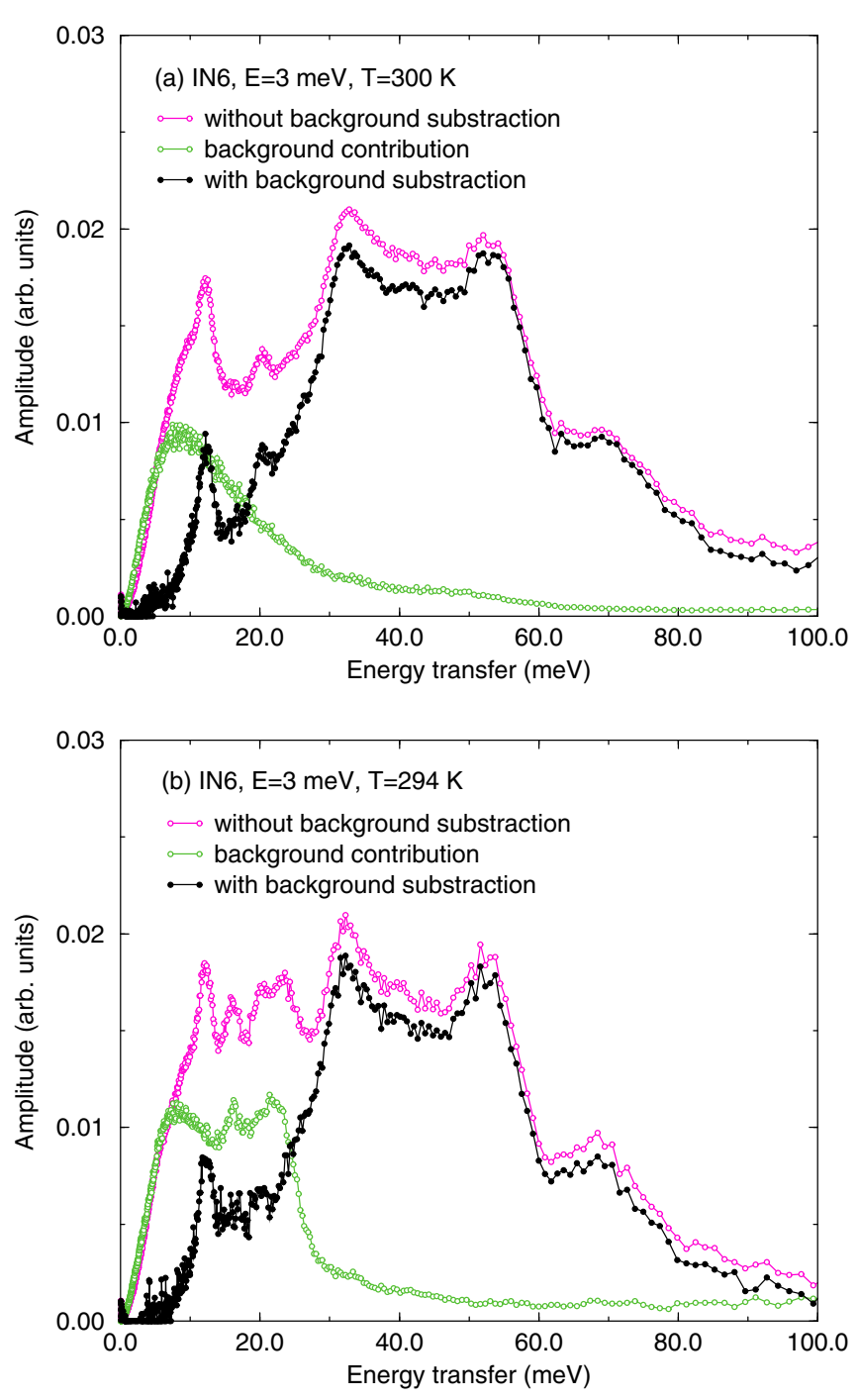

FIG. 3. Examples of background contributions at room temperature in the case of the neutron-weighted multiphonon spectrum of $\mathrm{UO}_{2}$ deduced from the IN6 data measured with a quartz tube in a thin niobium sample-holder tube (a) and a thick niobium sample-holder tube (b).

IN6 at $T=300 \mathrm{~K}$ and $T=294 \mathrm{~K}$. The non-negligible contribution of the vibration modes of niobium on the top of the phonon modes of the uranium atoms favors the use of a thin niobium sample-holder tube. Results obtained up to $1675 \mathrm{~K}$ are shown in Fig. 4. The vibration modes of $\mathrm{UO}_{2}$ are observed at energy positions which are in excellent agreement with the theoretical predictions reported in Fig. 1 at room temperature. Our results show a low temperature dependence of the phonon mode shift. An overall decrease of about $-2 \mathrm{meV}$ from 300 to $1675 \mathrm{~K}$ can be estimated from both the acoustic and optical phonon mode positions. It has to be noted that the dip between the TO1 and LO1 phonon branches cannot be observed around $30 \mathrm{meV}$ because of the too-broad response function of IN6. In addition, above $60 \mathrm{meV}$, the increasing broadening of the phonon linewidth due to the temperature makes it difficult to accurately determine the peak position corresponding to the highest longitudinal optical phonons.

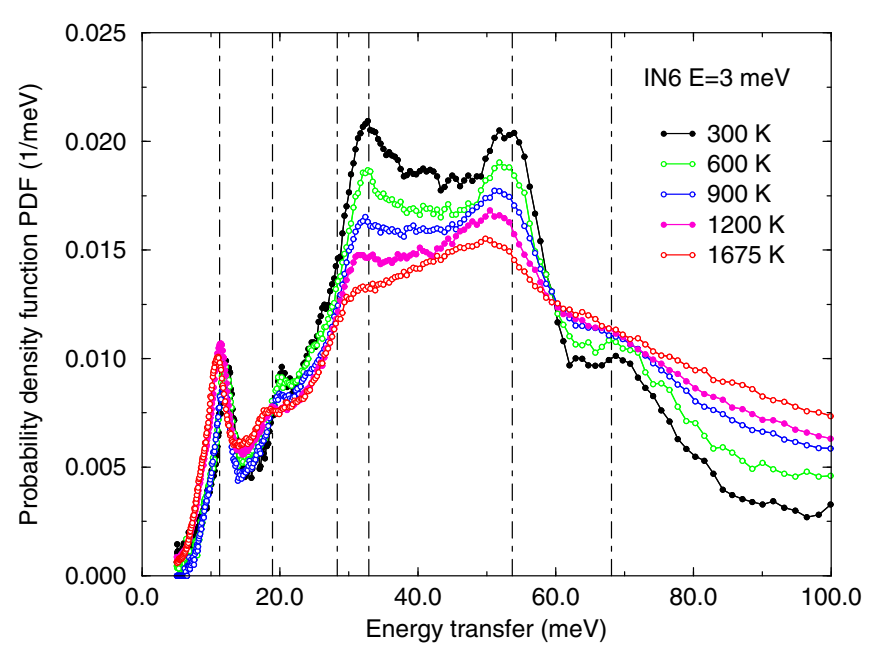

FIG. 4. Experimental neutron-weighted multiphonon spectrum $\rho_{\text {exp }}^{T}$ of $\mathrm{UO}_{2}$ deduced from the IN6 data. The dot-dashed lines show the energy positions of the vibration modes of $\mathrm{UO}_{2}$ calculated at $T=294$ K (Fig. 1).

\section{MONTE CARLO NEUTRON TRANSPORT SIMULATIONS}

Experimental neutron-weighted multiphonon spectrum $\rho_{\exp }^{T}$ of $\mathrm{UO}_{2}$ can only be compared with their theoretical analogs $\rho_{\text {th }}^{T}$ via Monte Carlo simulations that take into account the multiple neutron scattering effects and multiphonon contribution. In this work, the time of flight $t$ of each neutron has been simulated with the neutron transport code TRIPOLI4 without taking into account the time distribution of the initial neutron burst originating from the Fermi chopper. Therefore, the theoretical neutron yield $Y_{\text {th }}^{T}$ is calculated from the neutron yields $Y_{T 4}^{T}$ provided by TRIPOLI4 as follows:

$$
Y_{\text {th }}^{T}\left(\theta, E^{\prime}\right)=\int_{0}^{+\infty} R_{E^{\prime}}(\theta, t) Y_{T 4}^{T}(\theta, t) d t .
$$

The probability density function $R_{E^{\prime}}(\theta, t)$ stands for the time-dependent experimental response function of the spectrometer which is well approximated by a Gaussian. Its full width at half maximum $\Delta t=41(5) \mu \mathrm{s}$ (for IN6 at $E=$ $3 \mathrm{meV}$ ) and $\Delta t=22(5) \mu \mathrm{s}$ (for IN4 at $E=112 \mathrm{meV}$ ) was fitted to the neutron elastic scattering peak of vanadium.

Figure 5 compares the experimental and theoretical neutron-weighted multiphonon spectra obtained in the case of the IN6 spectrometer at $T=294 \mathrm{~K}$ when the $a b$ initio phonon density of sates established at the Uppsala University (Fig. 1) are introduced in the TRIPOLI4 calculations. The top and middle plots illustrate the poor description of the experimental values when the single neutron scattering and one-phonon approximations are used. An overall agreement is achieved between the experimental and theoretical curves only if multiple scattering and multiphonon effects are taken into account in the simulation. The amplitude and the energy position of the vibration modes of the $\mathrm{UO}_{2}$ molecule are well predicted confirming the validity of the main approximations involved in the $a b$ initio calculations (quasiharmonic phonon-based model), neutron scattering formalism (inelastic and Gaussian approximations), Monte Carlo simulations (Gaussian response function), and data reduction procedure 

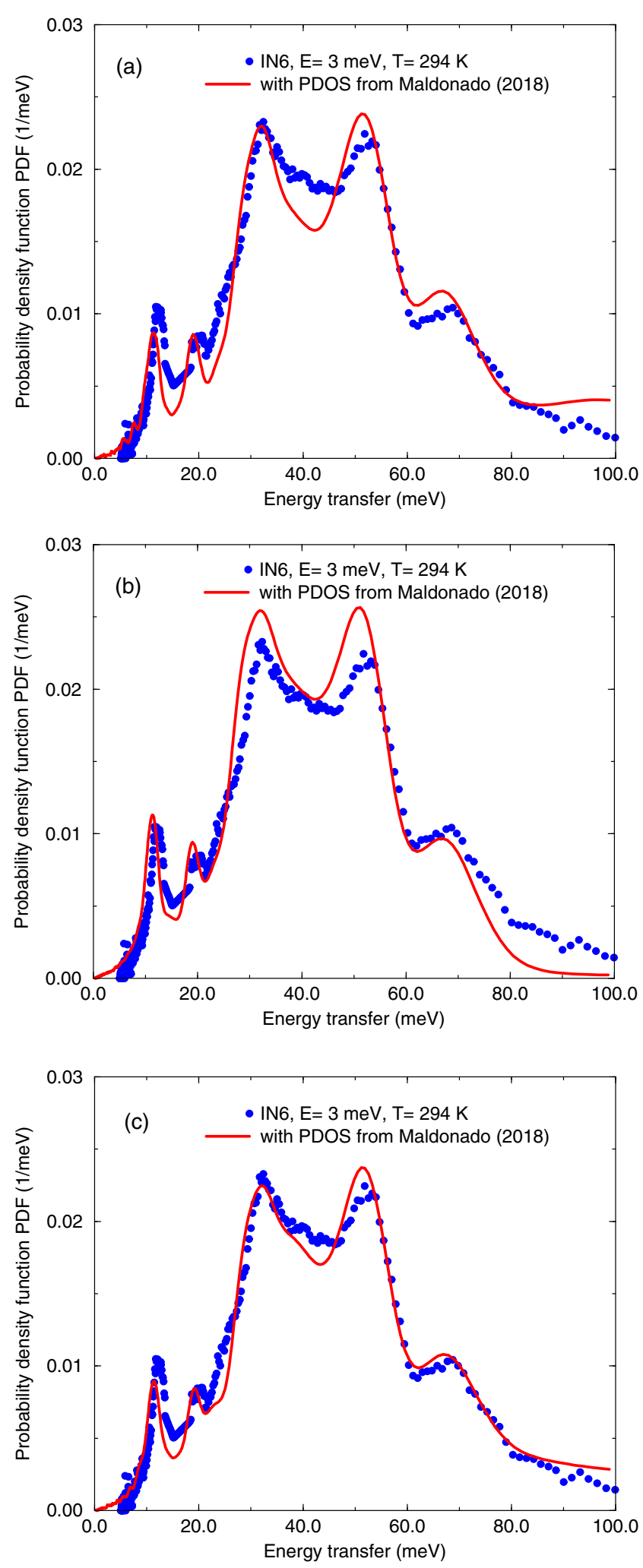

FIG. 5. Comparison of the neutron-weighted multiphonon spectrum $\rho_{\exp }^{T}$ and $\rho_{\text {th }}^{T}$ deduced from the IN6 data and the TRIPOLI4 simulations, respectively. The calculations were performed at $T=294 \mathrm{~K}$ by using the PDOS shown in Fig. 1 . The theoretical curves in plots (a) and (b) were obtained within the single neutron scattering and one-phonon approximations, respectively. The theoretical curve in the bottom plot (c) accounts for both the multiple neutron scattering effects and the multiphonon contribution. (empty-cell subtraction strategy and extrapolation of the cumulative distribution function of $P_{\exp }^{T}(\alpha, \beta)$ to $\left.\alpha=0\right)$. Despite the achievement observed at room temperature, moving toward higher temperatures with ab initio simulations represents a theoretical challenge because anharmonicity may cause sizable interactions among phonons with increasing temperature. Even if anharmonic effects in neutron scattering by crystalline materials were the subject of numerical calculations from the early 1960s [24,25], they still remain an issue to study inelastic neutron scattering data.

\section{RESULTS AND DISCUSSIONS}

To work around such theoretical limitations, combining $a b$ initio PDOS and Monte Carlo inelastic neutron scattering calculations in a Bayesian learning framework can be an alternative approach to assess temperature-dependent PDOS for improving comparison to experiment and exploring first-principles calculation hypothesis. In the present work, the phonon density of states $\rho_{X}^{T}$ of uranium and oxygen in $\mathrm{UO}_{2}$ were optimized on the IN6 data with an iterative Bayesian least-squares fitting procedure. Best estimates of the phonon density of states were found thanks to the chi-square minimization capabilities implemented in the nuclear data code CONRAD [26]. Starting from the PDOS of Wormald et al. (Fig. 1), our model gradually converged towards the posterior solutions shown in Fig. 6 for 300, 350, 420, 600, 900,1200 , and $1675 \mathrm{~K}$, leading to a good agreement between the posterior TRIPOLI4 simulations and the experimental symmetric form of the dynamic structure factor $S_{\text {exp }}^{T}$, in which the quasielastic neutron scattering peak has been removed [Eq. (9)]. An example of results is shown in Fig. 7 for $\theta=90^{\circ}$. Most of the differences between the prior calculations and the data have been reduced, especially around $30 \mathrm{meV}$. This energy range corresponds to the TO1/LO1 splitting which is softened by the response function of the IN6 spectrometer. This result agrees with the conclusions already reported by Pang et al. [12] about the contribution of the optical phonons to the thermal conductivity of $\mathrm{UO}_{2}$ and the need for improving the theoretical description of the phonon broadening near $30 \mathrm{meV}$, where the contribution of the uranium atoms is still important.

The optimized partial phonon density of states $\rho_{X}^{T}$ of uranium $\left(X={ }^{238} \mathrm{U}\right)$ and oxygen $\left(X={ }^{16} \mathrm{O}\right)$ in $\mathrm{UO}_{2}$ are shown in Fig. 8 as a function of the temperature. The corresponding values are reported in the Appendix (Tables I and II). As expected from Fig. 4, the energy shift of the acoustic and optical phonon modes is close to $-2 \mathrm{meV}$ on average from 300 to $1675 \mathrm{~K}$, corresponding to a phonon frequency shift $\omega_{1675 \mathrm{~K}}-\omega_{300 \mathrm{~K}}=-0.4 \mathrm{THz}$. This order of magnitude is compatible with those obtained via models involving fourphonon scattering [27]. No meaningful anomaly is observed in the increasing broadening of the acoustic and optical phonon linewidths. The phonon linewidths corresponding to the TO2 $(\varepsilon \simeq 55 \mathrm{meV})$ and LO2 $(\varepsilon \simeq 70 \mathrm{meV})$ phonon branches increase by a factor of about 1.5 from 300 to $1675 \mathrm{~K}$. As already observed by Hutchings [28], the broadening of the acoustic modes seems to be less important. Similarly, methods relying on interacting phonon theory, already applied over the last 10 years to investigate the anharmonicity-induced phonon broadening, should be able to explain part of the monotonic 

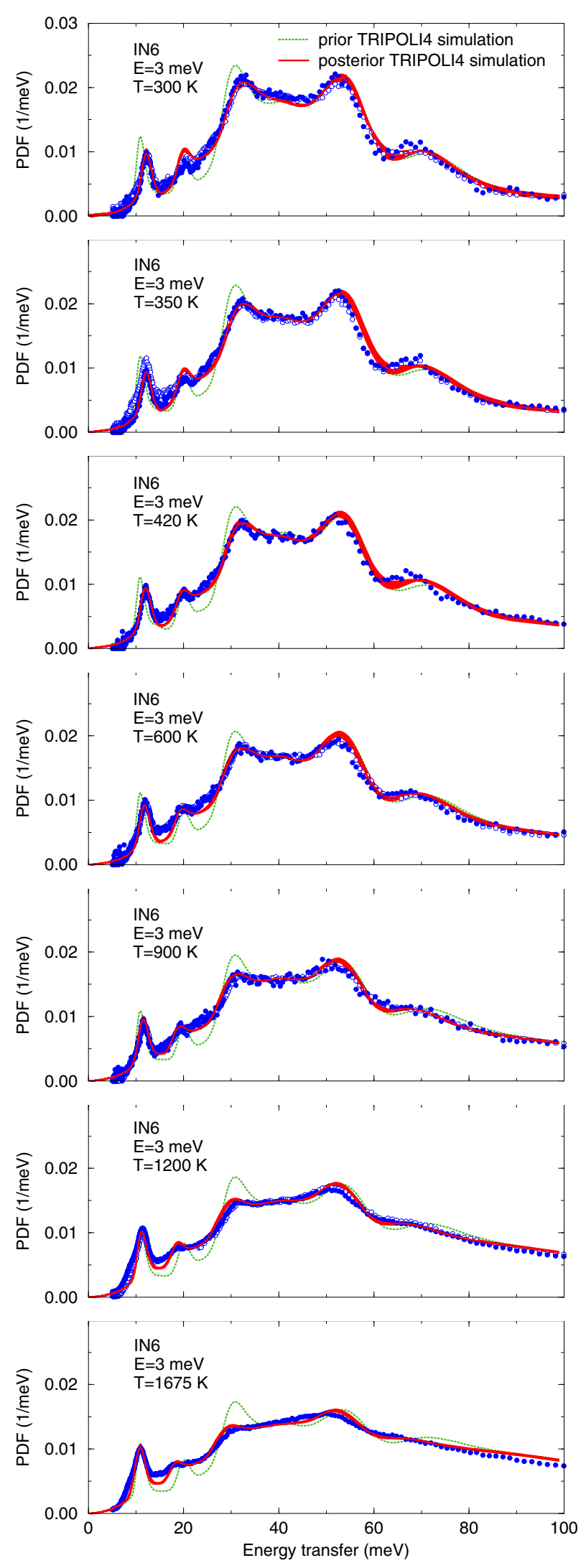

FIG. 6. Prior and posterior TRIPOLI4 simulations compared to the neutron-weighted multiphonon spectra deduced from the IN6 data.

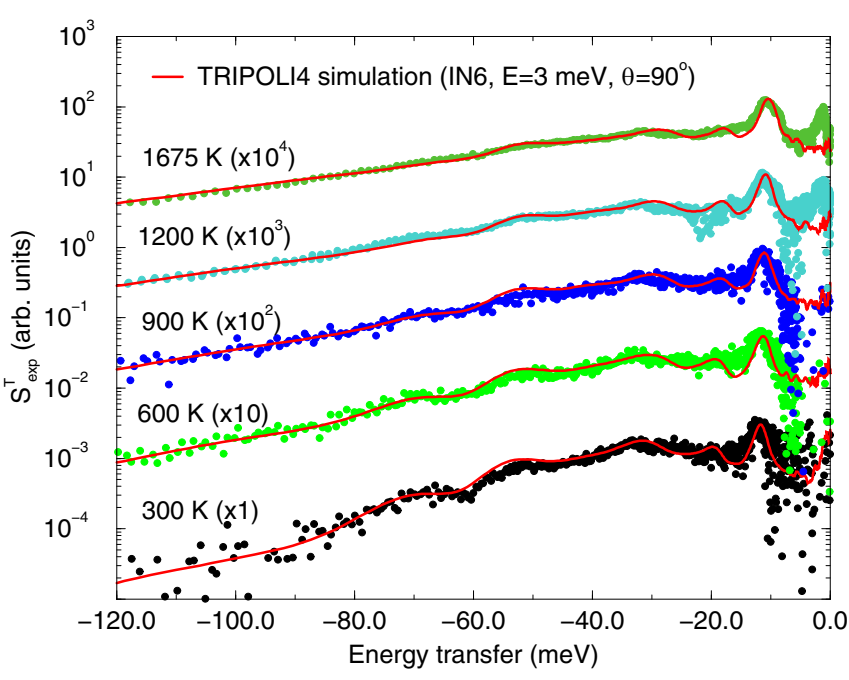

FIG. 7. Experimental symmetric form of the dynamic structure factor $S_{\exp }^{T}$ without the contribution of the quasielastic neutron scattering peak [Eq. (9)] measured at $\theta=90^{\circ}$ with the IN6 spectrometer. The solid red lines correspond to the posterior TRIPOLI4 simulations obtained after the optimization of the partial phonon density of states $\rho_{X}^{T}$ of uranium and oxygen in $\mathrm{UO}_{2}$.

behavior of the phonon linewidth observed as a function of the temperature.

The higher incident neutron energy range was investigated with the IN4 spectrometer by using a monoenergetic neutron beam $E=112 \mathrm{meV}$. Although the poor response function of IN4 is not suited to study the fine phonon structures of $\mathrm{UO}_{2}$, the spectrometer allows one to investigate the shape of the neutron-weighted multiphonon spectrum in the up- and down-scattering energy ranges. For that purpose, TRIPOLI4 simulations were repeated by using the IN4 model shown in Fig. 2 together with the partial PDOS deduced from the IN6 data (Fig. 8). Results obtained at 300, 600, and $900 \mathrm{~K}$ are

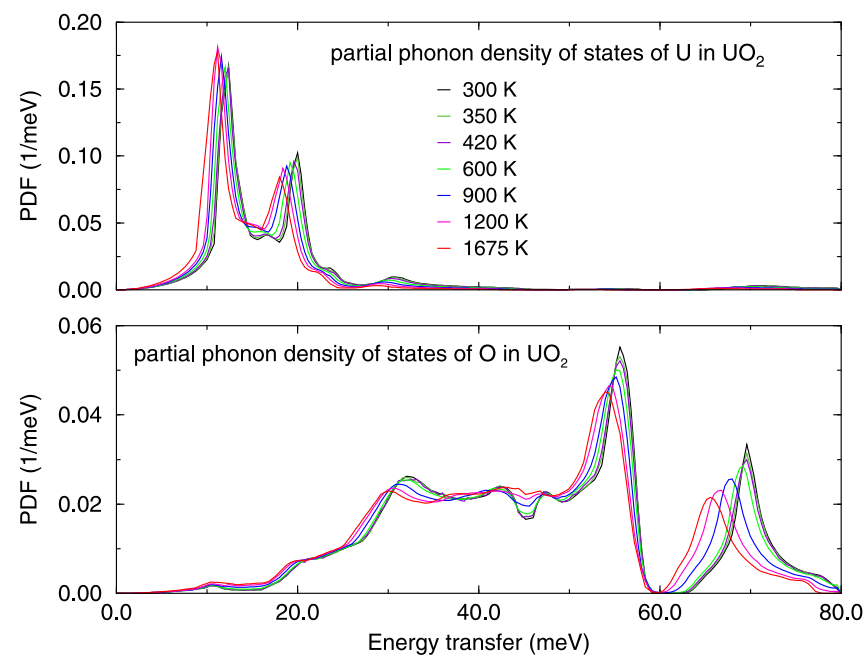

FIG. 8. Temperature-dependent partial phonon density of states $\rho_{X}^{T}$ of uranium and oxygen in $\mathrm{UO}_{2}$ deduced from the IN6 data. The corresponding values are reported in the Appendix (Tables I and II). 


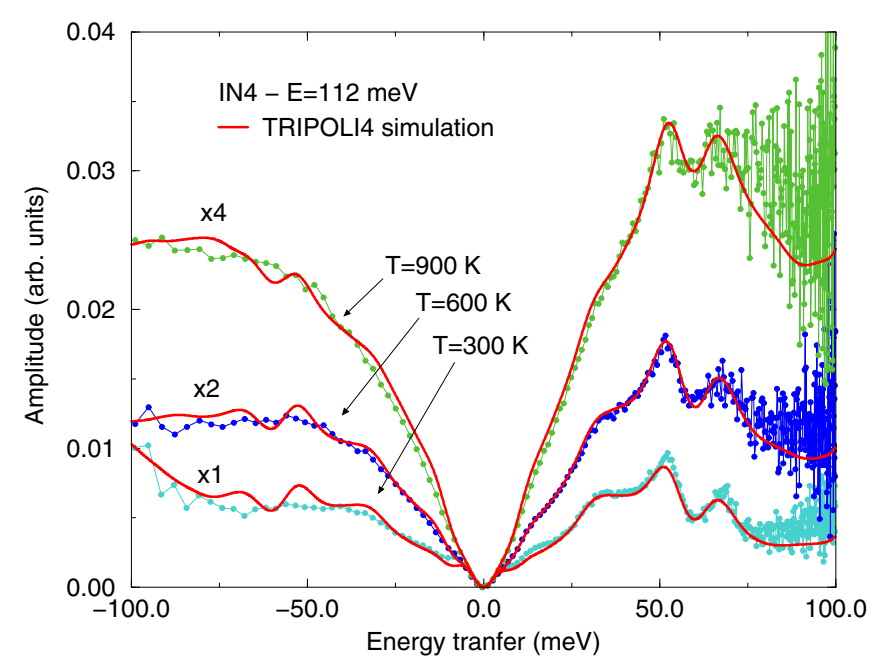

FIG. 9. Comparison of the neutron-weighted multiphonon spectra $\rho_{\exp }^{T}$ and $\rho_{\text {th }}^{T}$ deduced from the IN4 data and the TRIPOLI4 simulations, respectively. The calculations were performed at 300 , 600 , and $900 \mathrm{~K}$ by using the partial PDOS obtained from the IN6 data (Fig. 8).

shown in Fig. 9 as a function of the energy transfer ranging from -100 to $100 \mathrm{meV}$. The overall agreement achieved between the IN4 data and the Monte Carlo results validates the Bayesian inference method, which is presented in this work as an alternative approach to assess temperature-dependent partial PDOS for uranium and oxygen in $\mathrm{UO}_{2}$ starting from ab initio information.

The results inferred from the interpretation of the IN4 and IN6 data evidence the smooth deviation from the quasiharmonic approximation of the PDOS characteristics as a function of the temperature. A part of this behavior can be illustrated via the underlying Debye-Waller coefficient $\lambda_{s}$ involved in Eq. (4), a measure of which is given by the B factor. This parameter, also called the temperature parameter, is reported in the literature in units of $\AA^{2}$. It gives a measure of the dynamical disorder of the material. At room temperature, a low B-factor value is expected since the amplitude of the atomic thermal motion being smaller than the dynamical disorder of atoms is reduced. The expression of the $\mathrm{B}$ factor $B_{X}(T)$ for the oxygen and uranium atoms is given by [29]

$$
B_{X}(T)=8 \pi^{2} \frac{\hbar^{2}}{2 M_{X}} \int_{0}^{+\infty} \frac{\rho_{X}^{T}(\varepsilon)}{\varepsilon} \operatorname{coth}\left(\frac{\varepsilon}{2 k_{B} T}\right) d \varepsilon .
$$

Figure 10 shows the $\mathrm{B}$ factors obtained with the partial PDOS $\rho_{X}^{T}$ deduced from the IN6 data. Our calculations adequately follow the trend of a few data sets retrieved from the literature. The $\mathrm{B}$ factor of the uranium atoms exhibits a nearly linear dependence with the temperature until $1200 \mathrm{~K}$, which is in favor of an increasing anharmonic relaxation of the oxygen atom positions in a fluorite structure [30]. At $1675 \mathrm{~K}$, the clear deviation from the linearity of $B_{U}$ and $B_{O}$ may confirm the existence of a more complex atomic disorder with the distortion of the oxygen and uranium sublattices [31,32].

\section{CONCLUSIONS}

The IN4 and IN6 time-of-flight spectrometers of ILL were used to determine the main features of the partial phonon den-

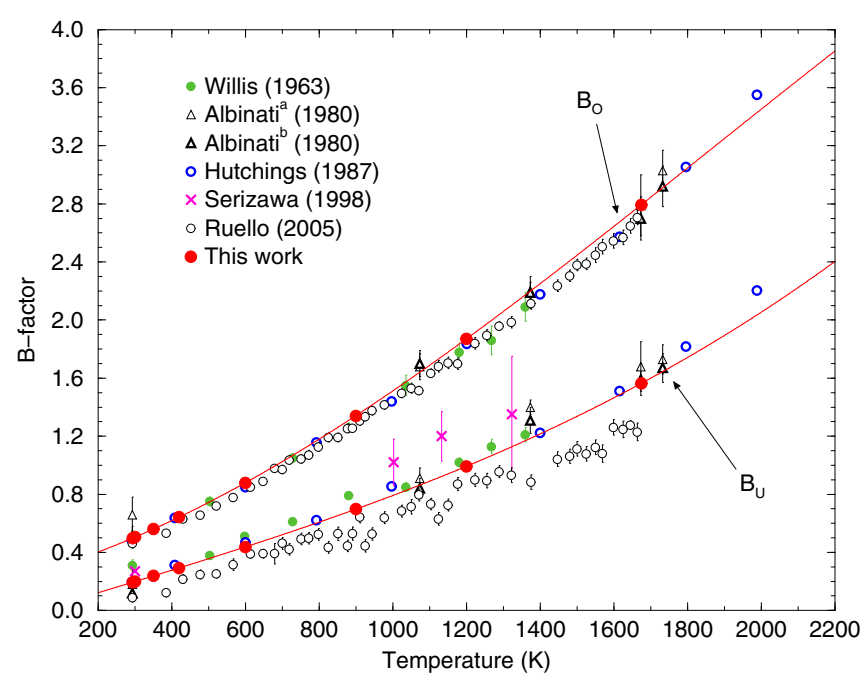

FIG. 10. The red filled circles (connected by the red "eye-guide" curves) represent the B factors (in $\AA^{2}$ ) calculated with Eq. (11) by using the partial PDOS obtained from the IN6 data (Fig. 8). They are compared with experimental values reported in Refs. [28,33-37].

sity of states of uranium and oxygen in $\mathrm{UO}_{2}$ as a function of the temperature. At room temperature, the neutron-weighted multiphonon spectra of $\mathrm{UO}_{2}$ derived from the inelastic neutron scattering data of IN6 is adequately reproduced by Monte Carlo neutron transport simulations relying on ab initio PDOS calculated at the Uppsala University. This result confirms the validity of the main approximations involved in the $a b$ initio calculations, neutron scattering formalism, Monte-Carlo simulations, and data reduction procedure.

For mapping the full temperature range of interest for the nuclear applications, a consistent set of PDOS was calculated within a Bayesian fitting framework starting from $a b$ initio information established by the Low Energy Interaction Physics group of the North Carolina State University. The posterior results confirm the need to improve the theoretical description of the TO1/LO1 splitting around $30 \mathrm{meV}$, where the contribution of the uranium atoms is still important. No anomalies with increasing temperatures were observed through the monotonic behavior of the phonon energy shift and of the phonon broadening. Our results also follow the behavior of the B-factor values, which smoothly increase with the temperature. The obtained PDOS can then be used to produce double-differential neutron scattering cross sections for simulating the neutron transport in UOX fuels from cold zero power to hot full power conditions.

In the temperature range between 294 and $1200 \mathrm{~K}$, the IN4 and IN6 data favor an increasing anharmonic relaxation of the oxygen atom positions in a fluorite structure. The models involving four-phonon scattering should be able to explain part of the observed monotonic behavior of the PDOS features. Above $1675 \mathrm{~K}$, the existence of a more complex atomic disorder with the distortion of the oxygen and uranium sublattices could be invoked to describe the experimental neutron-weighted multiphonon spectrum of $\mathrm{UO}_{2}$ measured at the ILL facilities. It has to be noted that temperatures close to $1700 \mathrm{~K}$ represent an upper limit for applying a Bayesian analysis to $\mathrm{UO}_{2}$ data measured on IN6 because the fine structures 
of the PDOS, which are already broadened by the temperature effects, are also too softened by the response function of the spectrometer. Exploring higher temperatures, closer to an accident scenario in power reactors, would require facilities with a smaller incident neutron burst width.

\section{ACKNOWLEDGMENTS}

The authors wish to express their appreciation for the experimental work performed at ILL with the specific support of F. Marchal and P. Mouveau for the sample preparation. Special thanks goes to G. Carlot and L. Desgrange, working in the Fuel Studies Department of CEA/Cadarache, for their valuable help during the preparation of this work. The Monte Carlo simulations were performed thanks to the relevant advice of C. Jouanne from CEA/Saclay. One of the authors also expresses his gratitude to Judy W. L. Pang for sharing her data for intercomparison studies.

\section{APPENDIX: PARTIAL PHONON DENSITY OF STATES OF URANIUM AND OXYGEN IN UO,}

Tables I and II report the temperature-dependent PDOS of uranium and oxygen in $\mathrm{UO}_{2}$ which are shown in Fig. 8. They were inferred from the IN6 data with the iterative Bayesian least-squares fitting procedure implemented in the nuclear data code CONRAD [26].

TABLE I. Partial phonon density of states of uranium in $\mathrm{UO}_{2}$ (in $1 / \mathrm{eV}$ ) as a function of the energy transfer (in $\mathrm{meV}$ ).

\begin{tabular}{|c|c|c|c|c|c|c|c|}
\hline$\varepsilon$ & $300 \mathrm{~K}$ & $350 \mathrm{~K}$ & $420 \mathrm{~K}$ & $600 \mathrm{~K}$ & $900 \mathrm{~K}$ & $1200 \mathrm{~K}$ & $1675 \mathrm{~K}$ \\
\hline 0.0 & 0.00 & 0.00 & 0.00 & 0.00 & 0.00 & .00 & 0.00 \\
\hline 0.4 & 0.04 & 0.04 & 0.05 & 0.05 & 0.05 & 6 & 0.07 \\
\hline 0.8 & 0.09 & 0.10 & 0.11 & 0.12 & 0.14 & 14 & 0.17 \\
\hline 1.2 & 0.21 & 0.22 & 0.23 & 0.25 & 0.27 & 0.29 & 0.35 \\
\hline 1.6 & 0.34 & 0.36 & 0.38 & 0.41 & & 0.48 & 0.60 \\
\hline 2.0 & 0.54 & 0.56 & 0.58 & 0.62 & 0.68 & 73 & 0.92 \\
\hline 2.4 & 0.76 & 0.79 & 0.81 & 0.88 & 0.95 & 1.03 & 1.32 \\
\hline 2.8 & 1.04 & 1.06 & 1.09 & & 1.27 & & 1.76 \\
\hline 3.2 & 1.34 & 1.38 & 1.42 & & 1.67 & & 2.34 \\
\hline 3.6 & 1.72 & 1.76 & 1.80 & 1.95 & 2.14 & 2.32 & 2.98 \\
\hline 4.0 & 2.13 & 2.19 & 2. & 4 & & 1 & 3.75 \\
\hline 4.4 & 2.63 & 2.69 & 2.76 & 2. & 3.28 & & 4.61 \\
\hline 4.8 & 3.16 & 3.24 & 3.32 & 3.60 & 3.96 & 1 & 5.54 \\
\hline 5.2 & 3.76 & 3.85 & 3.94 & 4.28 & 4.70 & 2 & 6.62 \\
\hline 5.6 & 4.41 & 4.52 & 4.63 & 5 & & 2 & 7.77 \\
\hline 6.0 & 5.13 & 5.26 & 5.40 & 5.8 & 6. & 7.08 & 9.20 \\
\hline 6.4 & 5.96 & 6.11 & 6.27 & 6.84 & 7.55 & 8.28 & 10.77 \\
\hline 6.8 & 6.88 & 7.07 & 7.26 & 7. & 8 & 57 & 12.60 \\
\hline 7.2 & 7.95 & 8.15 & 8.38 & 9.20 & 10.23 & 11.32 & 14.85 \\
\hline 7.6 & 9.13 & 9.41 & 9.69 & 10.67 & 4 & 13.26 & 17.38 \\
\hline 8.0 & 10.59 & 10.89 & 3 & 2 & & & 20.66 \\
\hline 8.4 & 12.22 & 12.62 & 13.02 & 14. & 16. & 18.31 & 24.28 \\
\hline 8.8 & 14.24 & 14.66 & 15.17 & 16.92 & 19.25 & 21.77 & 29.31 \\
\hline 9.2 & 16.44 & 17.04 & 17.72 & 19.98 & 23.00 & 26.41 & 57.94 \\
\hline 9.6 & 19.42 & 20.07 & 20.99 & 23.88 & 28.17 & 32.87 & 86.58 \\
\hline 10.0 & 22.67 & 23.84 & 25.49 & 30.13 & 38.80 & 54.27 & 115.21 \\
\hline 10.4 & 28.32 & 30.00 & 33.61 & 43.52 & 70.60 & 106.01 & 143.85 \\
\hline 10.8 & 34.53 & 43.29 & 54.88 & 82.52 & 119.74 & & 168.72 \\
\hline 11.2 & 79.97 & 88.46 & 99.39 & 128.41 & 151.31 & 181.70 & 178.35 \\
\hline 11.6 & 131.02 & 133.48 & 136.45 & 151.63 & 174.24 & 168.26 & 133.20 \\
\hline 12.0 & 150.29 & 151.72 & 159.85 & 166.04 & 149.31 & 128.01 & 97.96 \\
\hline
\end{tabular}

TABLE I. (Continued.)

\begin{tabular}{|c|c|c|c|c|c|c|c|}
\hline$\varepsilon$ & $300 \mathrm{~K}$ & $350 \mathrm{~K}$ & $420 \mathrm{~K}$ & $600 \mathrm{~K}$ & $900 \mathrm{~K}$ & $1200 \mathrm{~K}$ & $1675 \mathrm{~K}$ \\
\hline 12.4 & 167.45 & 166.22 & 165.31 & 135.43 & 16.40 & 1 & 1 \\
\hline 12.8 & 31.03 & 125.79 & 118.44 & 104.97 & & & \\
\hline 13.2 & 95.81 & 96.22 & 92.21 & 82.33 & 69.63 & & 53.72 \\
\hline 13.6 & 78.82 & 76.86 & 73.23 & & & & 51.88 \\
\hline 14.0 & 62.46 & 61.53 & 58.48 & 53.64 & & & 50.51 \\
\hline 14.4 & 50.56 & 49.55 & 47.96 & & & & 49.71 \\
\hline 14.8 & 40.53 & 41.82 & 42.21 & & 47.16 & & 48.78 \\
\hline 15.2 & 38.60 & 39.35 & 40.28 & 43.13 & 46.83 & & 47.69 \\
\hline 15.6 & 37.53 & & & & & & 46.25 \\
\hline 16.0 & 39.06 & 40.00 & & & & & \\
\hline 16.4 & 40.59 & & & & & & 50.79 \\
\hline 5.8 & 40.97 & & & & & & \\
\hline 7.2 & 39.49 & & & & & & \\
\hline 17.6 & 37.67 & 37.70 & 38.34 & 12 & 56.02 & & 75.69 \\
\hline 80 & 35.67 & 37.55 & 40.31 & 50.10 & & & \\
\hline 3.4 & 39.19 & 42 & & & & & .03 \\
\hline 18.8 & 47.29 & 55.58 & 65.27 & & & & 67.41 \\
\hline 9.2 & & & & & & & \\
\hline 9.6 & 92.72 & 08 & & & & & 39.30 \\
\hline 20.0 & 102.41 & & & & & & 8.56 \\
\hline 0.4 & 86.46 & 78 & 92 & 6 & & & 21.38 \\
\hline 0.8 & & 56 & & & & & \\
\hline .2 & 42.03 & 38.82 & & & & & 13 \\
\hline 1.6 & 27.10 & 26.61 & 10 & & & & .97 \\
\hline 2.0 & 21.75 & 21.36 & 20.34 & & & & 13.64 \\
\hline 4 & 18.21 & 1 & 17 & 14 & & & 12.60 \\
\hline 2 & 16.41 & & & & & & 10.21 \\
\hline .2 & 16 & 1 & & & & & 7.51 \\
\hline 3.6 & 16.43 & 14.40 & 13.65 & & & & 4.61 \\
\hline 4.0 & 14.50 & 13.67 & 12.67 & 10 & & 5.2 & 3.03 \\
\hline 1.4 & 12.01 & & & & & & 1.79 \\
\hline 4.8 & & & & 2 & & & 1.51 \\
\hline 5.2 & & & & & & & 1.36 \\
\hline & & & & & & & 38 \\
\hline & & & & & & & 1.46 \\
\hline 26.4 & & & & & & & 1.56 \\
\hline 5.8 & 400 & & 0 & 1 & 3 & 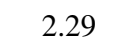 & 1.79 \\
\hline 7.2 & & & & & & & 2.08 \\
\hline 27.6 & 4.12 & 03 & 88 & 61 & 34 & 0 & 2.53 \\
\hline 28.0 & & & & & & & 2.92 \\
\hline & & & & & & & 23 \\
\hline & & & & & & & 3.25 \\
\hline 29.2 & & & & & & & 3.14 \\
\hline 29.6 & 48 & 2 & 1 & 9 & 2 & 1 & 2.81 \\
\hline 30.0 & & & & & & & 2.48 \\
\hline 30.4 & 50 & 91 & 41 & .19 & 5.33 & 6 & 2.16 \\
\hline & & & & & & & 1.91 \\
\hline 31.2 & & & & & & 2.84 & 1.74 \\
\hline 31.6 & & & & & & 2.57 & 1.60 \\
\hline 32.0 & 7.72 & & & & 3.36 & 2.36 & 1.47 \\
\hline 32.4 & 6.78 & & 5.59 & 4.29 & 3.08 & 2.18 & 1.38 \\
\hline & & & & 3.92 & & 2.03 & 1.29 \\
\hline 33.2 & 5.43 & 5.08 & 4.61 & 3.62 & 2.65 & 1.90 & 1.21 \\
\hline & & & & 3.37 & 2.48 & 1.79 & 1.14 \\
\hline 34.0 & 4.61 & 4.34 & 3.95 & $5.1 J$ & 2.33 & 1.68 & 1.07 \\
\hline 34.4 & 4.31 & & 3.70 & 2.96 & 2.20 & 1.59 & 1.02 \\
\hline & & & & 2.78 & 2.07 & 1.50 & 0.96 \\
\hline 35.2 & 3.79 & 3.57 & 3.27 & 2.63 & 1.96 & 1.43 & 0.92 \\
\hline 35.6 & 3.57 & 3.37 & 3.09 & 2.49 & 1.86 & 1.36 & 0.88 \\
\hline
\end{tabular}


TABLE I. (Continued.)

\begin{tabular}{|c|c|c|c|c|c|c|c|}
\hline$\varepsilon$ & $300 \mathrm{~K}$ & $350 \mathrm{~K}$ & $420 \mathrm{~K}$ & $600 \mathrm{~K}$ & $900 \mathrm{~K}$ & $1200 \mathrm{~K}$ & $1675 \mathrm{~K}$ \\
\hline 36.0 & 3.38 & 3.19 & 2.92 & 2.36 & 1.78 & 1.30 & 0.84 \\
\hline 36.4 & 3.20 & 3.02 & 2.77 & 2.25 & 1.70 & 1.24 & 0.81 \\
\hline 36.8 & 3.03 & 2.87 & 2.64 & 2.15 & 1.62 & 1.19 & 0.77 \\
\hline 37.2 & 2.89 & 2.74 & 2.52 & 2.06 & 1.56 & 1.14 & 0.75 \\
\hline 37.6 & 2.76 & 2.62 & 2.41 & 1.97 & 1.49 & 1.10 & 0.72 \\
\hline 38.0 & 2.65 & 2.51 & 2.31 & 1.89 & 1.44 & 1.07 & 0.69 \\
\hline 38.4 & 2.54 & 2.41 & 2.22 & 1.82 & 1.39 & 1.03 & 0.67 \\
\hline 38.8 & 2.44 & 2.31 & 2.13 & 1.76 & 1.34 & 0.99 & 0.64 \\
\hline 39.2 & 2.34 & 2.23 & 2.06 & 1.70 & 1.30 & 0.95 & 0.61 \\
\hline 39.6 & 2.26 & 2.15 & 1.99 & 1.64 & 1.25 & 0.91 & 0.58 \\
\hline 40.0 & 2.19 & 2.08 & 1.93 & 1.58 & 1.20 & 0.87 & 0.54 \\
\hline 40.4 & 2.12 & 2.01 & 1.86 & 1.52 & 1.15 & 0.82 & 0.50 \\
\hline 40.8 & 2.05 & 1.94 & 1.79 & 1.47 & 1.09 & 0.76 & 0.47 \\
\hline 41.2 & 1.97 & 1.87 & 1.73 & 1.40 & 1.02 & 0.71 & 0.42 \\
\hline 41.6 & 1.90 & 1.80 & 1.66 & 1.33 & 0.95 & 0.65 & 0.34 \\
\hline 42.0 & 1.83 & 1.73 & 1.58 & 1.24 & 0.89 & 0.56 & 0.27 \\
\hline 42.4 & 1.75 & 1.64 & 1.48 & 1.16 & 0.80 & 0.45 & 0.19 \\
\hline 42.8 & 1.63 & 1.53 & 1.38 & 1.07 & 0.68 & 0.33 & 0.15 \\
\hline 43.2 & 1.52 & 1.43 & 1.30 & 0.95 & 0.52 & 0.25 & 0.13 \\
\hline 43.6 & 1.44 & 1.33 & 1.18 & 0.79 & 0.38 & 0.20 & 0.11 \\
\hline 44.0 & 1.32 & 1.19 & 1.02 & 0.60 & 0.30 & 0.18 & 0.10 \\
\hline 44.4 & 1.15 & 1.00 & 0.81 & 0.45 & 0.25 & 0.16 & 0.09 \\
\hline 44.8 & 0.91 & 0.76 & 0.58 & 0.35 & 0.22 & 0.14 & 0.07 \\
\hline 45.2 & 0.63 & 0.56 & 0.46 & 0.30 & 0.19 & 0.12 & 0.07 \\
\hline 45.6 & 0.50 & 0.44 & 0.37 & 0.27 & 0.17 & 0.10 & 0.06 \\
\hline 46.0 & 0.40 & 0.37 & 0.33 & 0.24 & 0.15 & 0.09 & 0.06 \\
\hline 46.4 & 0.37 & 0.34 & 0.29 & 0.21 & 0.13 & 0.09 & 0.07 \\
\hline 46.8 & 0.33 & 0.30 & 0.26 & 0.18 & 0.12 & 0.09 & 0.07 \\
\hline 47.2 & 0.29 & 0.26 & 0.23 & 0.16 & 0.12 & 0.10 & 0.08 \\
\hline 47.6 & 0.25 & 0.23 & 0.20 & 0.15 & 0.12 & 0.10 & 0.09 \\
\hline 48.0 & 0.22 & 0.20 & 0.18 & 0.14 & 0.13 & 0.11 & 0.10 \\
\hline 48.4 & 0.20 & 0.18 & 0.17 & 0.15 & 0.14 & 0.13 & 0.11 \\
\hline 48.8 & 0.18 & 0.18 & 0.17 & 0.16 & 0.15 & 0.14 & 0.12 \\
\hline 49.2 & 0.18 & 0.18 & 0.17 & 0.17 & 0.17 & 0.16 & 0.14 \\
\hline 49.6 & 0.19 & 0.19 & 0.19 & 0.18 & 0.19 & 0.18 & 0.15 \\
\hline 50.0 & 0.20 & 0.20 & 0.20 & 0.20 & 0.21 & 0.20 & 0.17 \\
\hline 50.4 & 0.22 & 0.22 & 0.22 & 0.23 & 0.23 & 0.22 & 0.19 \\
\hline 50.8 & 0.24 & 0.24 & 0.25 & 0.26 & 0.26 & 0.25 & 0.21 \\
\hline 51.2 & 0.27 & 0.27 & 0.27 & 0.29 & 0.29 & 0.27 & 0.23 \\
\hline 51.6 & 0.30 & 0.30 & 0.31 & 0.32 & 0.32 & 0.30 & 0.26 \\
\hline 52.0 & 0.33 & 0.34 & 0.34 & 0.35 & 0.35 & 0.34 & 0.29 \\
\hline 52.4 & 0.37 & 0.37 & 0.38 & 0.39 & 0.38 & 0.38 & 0.31 \\
\hline 52.8 & 0.41 & 0.41 & 0.42 & 0.43 & 0.43 & 0.43 & 0.31 \\
\hline 53.2 & 0.45 & 0.46 & 0.46 & 0.47 & 0.49 & 0.45 & 0.26 \\
\hline 53.6 & 0.49 & 0.50 & 0.50 & 0.53 & 0.55 & 0.44 & 0.21 \\
\hline 54.0 & 0.54 & 0.55 & 0.56 & 0.59 & 0.58 & 0.39 & 0.12 \\
\hline 54.4 & 0.60 & 0.61 & 0.62 & 0.67 & 0.58 & 0.30 & 0.06 \\
\hline 54.8 & 0.66 & 0.68 & 0.71 & 0.71 & 0.52 & 0.20 & 0.02 \\
\hline 55.2 & 0.75 & 0.78 & 0.78 & 0.72 & 0.42 & 0.10 & 0.00 \\
\hline 55.6 & 0.86 & 0.85 & 0.83 & 0.67 & 0.28 & 0.04 & 0.00 \\
\hline 56.0 & 0.92 & 0.90 & 0.83 & 0.55 & 0.15 & 0.01 & 0.00 \\
\hline 56.4 & 0.96 & 0.88 & 0.76 & 0.39 & 0.06 & 0.00 & 0.00 \\
\hline 56.8 & 0.89 & 0.78 & 0.62 & 0.23 & 0.02 & 0.00 & 0.00 \\
\hline 57.2 & 0.76 & 0.61 & 0.43 & 0.09 & 0.00 & 0.00 & 0.00 \\
\hline 57.6 & 0.53 & 0.40 & 0.24 & 0.03 & 0.00 & 0.00 & 0.00 \\
\hline 58.0 & 0.31 & 0.19 & 0.10 & 0.01 & 0.00 & 0.00 & 0.00 \\
\hline
\end{tabular}

TABLE I. (Continued.)

\begin{tabular}{|c|c|c|c|c|c|c|c|}
\hline$\varepsilon$ & $300 \mathrm{~K}$ & $350 \mathrm{~K}$ & $420 \mathrm{~K}$ & $600 \mathrm{~K}$ & $900 \mathrm{~K}$ & $1200 \mathrm{~K}$ & $1675 \mathrm{~K}$ \\
\hline 58.4 & 0.09 & 0.07 & 0.03 & 0.00 & 0.00 & 0.00 & 0.00 \\
\hline 58.8 & 0.03 & 0.02 & 0.01 & 0.00 & 0.00 & 0.00 & 0.00 \\
\hline 59.2 & 0.00 & 0.00 & 0.00 & 0.00 & 0.00 & 0.00 & 0.00 \\
\hline 59.6 & 0.00 & 0.00 & 0.00 & 0.00 & 0.00 & 0.00 & 0.00 \\
\hline 60.0 & 0.00 & 0.00 & 0.00 & 0.00 & 0.00 & 0.00 & 0.01 \\
\hline 60.4 & 0.00 & 0.00 & 0.00 & 0.00 & 0.00 & 0.00 & 0.02 \\
\hline 60.8 & 0.00 & 0.00 & 0.00 & 0.00 & 0.00 & 0.01 & 0.04 \\
\hline 61.2 & 0.00 & 0.00 & 0.00 & 0.00 & 0.00 & 0.02 & 0.07 \\
\hline 61.6 & 0.00 & 0.00 & 0.00 & 0.00 & 0.00 & 0.04 & 0.12 \\
\hline 62.0 & 0.00 & 0.00 & 0.00 & 0.00 & 0.01 & 0.09 & 0.18 \\
\hline 62.4 & 0.00 & 0.00 & 0.00 & 0.00 & 0.04 & 0.15 & 0.26 \\
\hline 62.8 & 0.00 & 0.00 & 0.00 & 0.01 & 0.08 & 0.22 & 0.36 \\
\hline 63.2 & 0.00 & 0.00 & 0.00 & 0.03 & 0.15 & 0.32 & 0.48 \\
\hline 63.6 & 0.00 & 0.00 & 0.01 & 0.07 & 0.23 & 0.44 & 0.59 \\
\hline 64.0 & 0.00 & 0.01 & 0.03 & 0.13 & 0.34 & 0.60 & 0.70 \\
\hline 64.4 & 0.01 & 0.03 & 0.07 & 0.23 & 0.47 & 0.77 & 0.78 \\
\hline 64.8 & 0.04 & 0.08 & 0.14 & 0.34 & 0.65 & 0.94 & 0.86 \\
\hline 65.2 & 0.11 & 0.17 & 0.25 & 0.47 & 0.85 & 1.08 & 0.94 \\
\hline 65.6 & 0.22 & 0.28 & 0.37 & 0.66 & 1.07 & 1.18 & 1.01 \\
\hline 66.0 & 0.34 & 0.42 & 0.52 & 0.89 & 1.27 & 1.29 & 1.06 \\
\hline 66.4 & 0.49 & 0.57 & 0.72 & 1.15 & 1.43 & 1.41 & 1.05 \\
\hline 66.8 & 0.66 & 0.80 & 0.98 & 1.42 & 1.57 & 1.47 & 1.03 \\
\hline 67.2 & 0.94 & 1.08 & 1.28 & 1.64 & 1.70 & 1.50 & 1.01 \\
\hline 67.6 & 1.25 & 1.42 & 1.59 & 1.83 & 1.82 & 1.52 & 0.99 \\
\hline 68.0 & 1.63 & 1.75 & 1.86 & 1.99 & 1.90 & 1.50 & 0.95 \\
\hline 68.4 & 1.94 & 2.02 & 2.08 & 2.15 & 1.94 & 1.47 & 0.89 \\
\hline 68.8 & 2.21 & 2.24 & 2.25 & 2.30 & 1.95 & 1.43 & 0.82 \\
\hline 69.2 & 2.40 & 2.42 & 2.45 & 2.37 & 1.92 & 1.37 & 0.76 \\
\hline 69.6 & 2.58 & 2.63 & 2.65 & 2.43 & 1.88 & 1.30 & 0.70 \\
\hline 70.0 & 2.84 & 2.84 & 2.73 & 2.42 & 1.82 & 1.23 & 0.66 \\
\hline 70.4 & 3.05 & 2.93 & 2.81 & 2.37 & 1.75 & 1.16 & 0.62 \\
\hline 70.8 & 3.15 & 3.01 & 2.81 & 2.31 & 1.67 & 1.10 & 0.59 \\
\hline 71.2 & 3.14 & 2.98 & 2.75 & 2.25 & 1.59 & 1.04 & 0.57 \\
\hline 71.6 & 3.06 & 2.91 & 2.69 & 2.17 & 1.51 & 0.98 & 0.54 \\
\hline 72.0 & 3.00 & 2.85 & 2.63 & 2.07 & 1.42 & 0.93 & 0.53 \\
\hline 72.4 & 2.95 & 2.78 & 2.53 & 1.96 & 1.33 & 0.89 & 0.52 \\
\hline 72.8 & 2.85 & 2.67 & 2.41 & 1.83 & 1.26 & 0.86 & 0.52 \\
\hline 73.2 & 2.71 & 2.52 & 2.26 & 1.71 & 1.20 & 0.84 & 0.51 \\
\hline 73.6 & 2.51 & 2.34 & 2.10 & 1.61 & 1.16 & 0.82 & 0.49 \\
\hline 74.0 & 2.32 & 2.16 & 1.95 & 1.53 & 1.12 & 0.79 & 0.43 \\
\hline 74.4 & 2.14 & 2.01 & 1.83 & 1.46 & 1.07 & 0.72 & 0.35 \\
\hline 74.8 & 2.02 & 1.90 & 1.74 & 1.40 & 1.01 & 0.64 & 0.24 \\
\hline 75.2 & 1.91 & 1.81 & 1.66 & 1.33 & 0.93 & 0.54 & 0.13 \\
\hline 75.6 & 1.82 & 1.72 & 1.58 & 1.25 & 0.84 & 0.44 & 0.06 \\
\hline 76.0 & 1.73 & 1.64 & 1.50 & 1.16 & 0.75 & 0.37 & 0.01 \\
\hline 76.4 & 1.66 & 1.56 & 1.43 & 1.10 & 0.70 & 0.34 & 0.00 \\
\hline 76.8 & 1.60 & 1.51 & 1.37 & 1.05 & 0.67 & 0.33 & 0.00 \\
\hline 77.2 & 1.55 & 1.47 & 1.34 & 1.03 & 0.66 & 0.32 & 0.00 \\
\hline 77.6 & 1.54 & 1.45 & 1.32 & 1.02 & 0.66 & 0.32 & 0.00 \\
\hline 78.0 & 1.53 & 1.44 & 1.32 & 1.02 & 0.66 & 0.32 & 0.00 \\
\hline 78.4 & 1.54 & 1.44 & 1.31 & 1.01 & 0.64 & 0.32 & 0.00 \\
\hline 78.8 & 1.49 & 1.39 & 1.27 & 0.98 & 0.62 & 0.30 & 0.00 \\
\hline 79.2 & 1.39 & 1.34 & 1.23 & 0.94 & 0.61 & 0.30 & 0.00 \\
\hline 79.6 & 1.16 & 1.34 & 1.23 & 0.94 & 0.61 & 0.30 & 0.00 \\
\hline 80.0 & 0.00 & 0.00 & 0.00 & 0.00 & 0.00 & 0.00 & 0.00 \\
\hline
\end{tabular}


TABLE II. Partial phonon density of states of oxygen in $\mathrm{UO}_{2}$ (in $1 / \mathrm{eV}$ ) as a function of the energy transfer (in $\mathrm{meV}$ ).

\begin{tabular}{|c|c|c|c|c|c|c|c|}
\hline$\varepsilon$ & $300 \mathrm{~K}$ & $350 \mathrm{~K}$ & $420 \mathrm{~K}$ & $600 \mathrm{~K}$ & $900 \mathrm{~K}$ & $1200 \mathrm{~K}$ & $1675 \mathrm{~K}$ \\
\hline 0.0 & 0.00 & 0.00 & 0.00 & 0.00 & 0.00 & 0.00 & 0.00 \\
\hline 0.8 & 0.01 & 0.01 & 0.02 & 0.01 & 0.01 & 0.01 & 0.01 \\
\hline 1.2 & 0.02 & 0.02 & 0.02 & 0.02 & 0.02 & 0.02 & 0.02 \\
\hline 1.6 & 0.03 & 0.02 & 0.05 & 0.03 & 0.03 & 0.03 & 0.04 \\
\hline 2.0 & 0.05 & 0.06 & 0.08 & 0.05 & 0.05 & 0.05 & 0.06 \\
\hline 2.4 & 0.07 & 0.06 & 0.07 & 0.07 & 0.07 & 0.07 & 0.09 \\
\hline 2.8 & 0.09 & 0.12 & 0.11 & 0.09 & 0.09 & 0.10 & 0.12 \\
\hline 3.2 & 0.12 & 0.13 & 0.13 & 0.11 & 0.12 & 0.13 & 0.16 \\
\hline 3.6 & 0.15 & 0.17 & 0.15 & 0.14 & 0.15 & 0.16 & 0.21 \\
\hline 4.0 & 0.19 & 0.15 & 0.25 & 0.18 & 0.18 & 0.19 & 0.25 \\
\hline 4.4 & 0.23 & 0.24 & 0.28 & 0.21 & 0.22 & 0.24 & 0.31 \\
\hline 4.8 & 0.27 & 0.29 & 0.27 & 0.25 & 0.26 & 0.28 & 0.37 \\
\hline 5.2 & 0.31 & 0.34 & 0.34 & 0.29 & 0.31 & 0.33 & 0.43 \\
\hline 5.6 & 0.36 & 0.39 & 0.47 & 0.34 & 0.35 & 0.38 & 0.49 \\
\hline 6.0 & 0.41 & 0.44 & 0.44 & 0.39 & 0.40 & 0.43 & 0.56 \\
\hline 6.4 & 0.47 & 0.51 & 0.50 & 0.44 & 0.46 & 0.49 & 0.64 \\
\hline 6.8 & 0.53 & 0.57 & 0.53 & 0.49 & 0.52 & 0.55 & 0.73 \\
\hline 7.2 & 0.59 & 0.68 & 0.69 & 0.56 & 0.59 & 0.63 & 0.82 \\
\hline 7.6 & 0.67 & 0.64 & 0.71 & 0.63 & 0.66 & 0.70 & 0.93 \\
\hline 8.0 & 0.75 & 0.76 & 0.80 & 0.70 & 0.74 & 0.79 & 1.04 \\
\hline 8.4 & 0.83 & 0.86 & 0.93 & 0.78 & 0.82 & 0.88 & 1.16 \\
\hline 8.8 & 0.93 & 1.06 & 1.01 & 0.87 & 0.91 & 0.97 & 1.28 \\
\hline 9.2 & 1.03 & 1.15 & 1.17 & 1.09 & 1.17 & 1.28 & 1.60 \\
\hline 9.6 & 1.16 & 1.17 & 1.24 & 1.32 & 1.42 & 1.58 & 1.93 \\
\hline 10.0 & 1.40 & 1.54 & 1.49 & 1.54 & 1.68 & 1.88 & 2.26 \\
\hline 10.4 & 1.70 & 1.64 & 1.71 & 1.76 & 1.93 & 2.18 & 2.59 \\
\hline 10.8 & 1.68 & 1.88 & 1.68 & 1.71 & 1.89 & 2.13 & 2.51 \\
\hline 11.2 & 1.57 & 1.52 & 1.68 & 1.62 & 1.77 & 2.00 & 2.44 \\
\hline 11.6 & 1.21 & 1.25 & 1.33 & 1.36 & 1.59 & 1.86 & 2.38 \\
\hline 12.0 & 0.96 & 0.92 & 1.08 & 1.16 & 1.44 & 1.71 & 2.32 \\
\hline 12.4 & 0.85 & 0.89 & 0.95 & 1.10 & 1.55 & 1.97 & 2.26 \\
\hline 12.8 & 0.79 & 0.84 & 0.95 & 1.02 & 1.45 & 1.84 & 2.12 \\
\hline 13.2 & 0.76 & 0.79 & 0.88 & 0.97 & 1.38 & 1.75 & 2.00 \\
\hline 13.6 & 0.75 & 0.79 & 0.89 & 0.95 & 1.36 & 1.75 & 2.01 \\
\hline 14.0 & 0.74 & 0.80 & 0.80 & 0.95 & 1.37 & 1.77 & 2.04 \\
\hline 14.4 & 0.74 & 0.73 & 0.82 & 0.96 & 1.40 & 1.82 & 2.10 \\
\hline 14.8 & 0.75 & 0.72 & 0.78 & 0.98 & 1.43 & 1.87 & 2.16 \\
\hline 15.2 & 0.76 & 0.80 & 0.81 & 1.00 & 1.47 & 1.92 & 2.22 \\
\hline 15.6 & 0.77 & 0.81 & 0.91 & 1.05 & 1.54 & 1.98 & 2.28 \\
\hline 16.0 & 0.79 & 0.85 & 0.99 & 1.11 & 1.61 & 2.07 & 2.35 \\
\hline 16.4 & 1.16 & 1.25 & 1.29 & 1.36 & 1.76 & 2.20 & 2.41 \\
\hline 16.8 & 1.53 & 1.60 & 1.58 & 1.72 & 2.08 & 2.41 & 2.61 \\
\hline 17.2 & 1.89 & 1.93 & 2.11 & 2.09 & 2.52 & 2.93 & 3.20 \\
\hline 17.6 & 2.28 & 2.32 & 2.40 & 2.50 & 3.01 & 3.54 & 3.91 \\
\hline 18.0 & 2.68 & 2.77 & 2.85 & 3.01 & 3.62 & 4.26 & 4.74 \\
\hline 18.4 & 3.31 & 3.39 & 3.48 & 3.66 & 4.34 & 5.05 & 5.55 \\
\hline 18.8 & 4.00 & 3.91 & 4.11 & 4.41 & 5.14 & 5.85 & 6.34 \\
\hline 19.2 & 4.83 & 4.99 & 4.90 & 5.20 & 5.92 & 6.55 & 6.85 \\
\hline 19.6 & 5.64 & 5.89 & 5.67 & 5.95 & 6.61 & 7.03 & 7.24 \\
\hline 20.0 & 6.40 & 6.43 & 6.32 & 6.59 & 7.10 & 7.28 & 7.36 \\
\hline 20.4 & 7.01 & 7.18 & 7.10 & 7.09 & 7.38 & 7.48 & 7.37 \\
\hline 20.8 & 7.52 & 7.26 & 6.99 & 7.44 & 7.56 & 7.46 & 7.29 \\
\hline 21.2 & 7.74 & 7.60 & 7.77 & 7.65 & 7.61 & 7.50 & 7.54 \\
\hline 21.6 & 7.88 & 7.99 & 7.78 & 7.72 & 7.67 & 7.72 & 7.84 \\
\hline 22.0 & 7.82 & 7.62 & 7.95 & 7.82 & 7.84 & 8.01 & 8.15 \\
\hline 22.4 & 7.86 & 7.81 & 8.16 & 8.00 & 8.11 & 8.32 & 8.46 \\
\hline 22.8 & 8.10 & 8.12 & 7.91 & 8.23 & 8.42 & 8.64 & 8.77 \\
\hline 23.2 & 8.38 & 8.28 & 8.31 & 8.52 & 8.76 & 8.96 & 9.07 \\
\hline
\end{tabular}

TABLE II. (Continued.)

\begin{tabular}{|c|c|c|c|c|c|c|c|}
\hline$\varepsilon$ & $300 \mathrm{~K}$ & $350 \mathrm{~K}$ & $420 \mathrm{~K}$ & $600 \mathrm{~K}$ & $900 \mathrm{~K}$ & $1200 \mathrm{~K}$ & $1675 \mathrm{~K}$ \\
\hline 23.6 & 8.69 & 75 & 77 & 86 & 9 & & 38 \\
\hline 4.0 & 9.07 & 3.99 & & & & & 9.69 \\
\hline 4.4 & 9.45 & 9.24 & 9.69 & 9.54 & 9.73 & 9.89 & 10.00 \\
\hline 4.8 & 9.77 & 89 & 9.88 & 9.86 & 10.03 & 10.20 & 10.30 \\
\hline 5.2 & 10.09 & 10.28 & 10.32 & 10.17 & 10.34 & 10.57 & 10.93 \\
\hline 5.6 & 10.40 & 10.43 & 10.67 & 10.48 & 10.67 & & 12.06 \\
\hline 6.0 & 10.71 & 10.83 & 10.96 & 10.79 & 11.10 & 12.14 & 13.18 \\
\hline 26.4 & 11.03 & 11.17 & 11.08 & 11.13 & 11.84 & 13.26 & 14.30 \\
\hline 26.8 & 11.34 & 11.52 & 11.45 & 11.63 & 12.88 & 14 & 15.42 \\
\hline 7.2 & 11.76 & 11.65 & 12.27 & 12.50 & 14 & 15.61 & 16.55 \\
\hline 7.6 & 12.73 & 12.96 & 12.97 & 13.59 & 15 & & 17.67 \\
\hline 3.0 & 13.87 & 13.82 & 14.25 & 14.87 & 16 & & 18.79 \\
\hline 3.4 & 15.23 & & & & & & 19.92 \\
\hline 3.8 & 16.62 & 16.54 & 17.01 & 17.56 & & 20 & 21.03 \\
\hline 29.2 & 18.01 & 18.53 & 18.60 & 18.96 & & & 2.15 \\
\hline 29.6 & 19.48 & 19.36 & 19.48 & 20 & 2 & 2 & 22.64 \\
\hline 0.0 & 20.94 & 21.26 & 21.64 & 21.77 & 2 & & 22.98 \\
\hline 0.4 & 22.43 & 22.67 & 22.80 & 23.06 & & & 22.87 \\
\hline 0.8 & 23.76 & 23.91 & 24.06 & 16 & 2 & & 22.57 \\
\hline 1.2 & 24.95 & 24.86 & & & & & 22.07 \\
\hline 1.6 & 25.66 & 25.89 & 25.25 & 36 & 0 & 22 & 21.61 \\
\hline & & & & & & & \\
\hline .4 & 26.1 & 2 & 2 & & & & 0.91 \\
\hline 2.8 & 25.96 & 25.35 & 25.37 & 24 & 2 & & 20.69 \\
\hline 3.2 & 25.51 & 24.90 & 25.60 & 5 & & & 20.54 \\
\hline 3.6 & 24.94 & 24.93 & 74 & 3 & 5 & 4 & 20.39 \\
\hline 4.0 & 24.27 & 24.18 & 23.77 & 2 & 5 & 6 & 20.24 \\
\hline 34.4 & 23.6 & 23.5 & 23.41 & 22.76 & 19 & 62 & 0.27 \\
\hline 4.8 & 23.03 & 23.01 & 22.66 & 22. & & & 36 \\
\hline 35.2 & 22.74 & 22.42 & 22.86 & 22 & 2 & & 20.73 \\
\hline 35.6 & 22.46 & 22.19 & 22.01 & 21 & & 20 & 21.11 \\
\hline 6.0 & 22.18 & 22.21 & 22.56 & 8 & & & 21.54 \\
\hline 36.4 & 21.90 & 21 & 21.25 & 6 & 9 & & 21.89 \\
\hline 36.8 & 21.63 & 21.54 & 21.48 & 21.19 & 1 & 0 & 22.18 \\
\hline 37.2 & 21.42 & 21.36 & 21.20 & 21.11 & & & 2.30 \\
\hline 6 & 21.25 & & & 6 & & & \\
\hline 3.0 & 21.19 & 21.29 & 20.86 & 5 & & 22 & 2.32 \\
\hline 8.4 & 21.15 & 20.96 & 20.67 & 21 & 2 & & 22.27 \\
\hline 38.8 & 21.18 & 21.51 & 21.40 & 21.24 & 95 & 9 & 22.23 \\
\hline 39.2 & 21.25 & 21.30 & 21.07 & 21.42 & 22.12 & 22.48 & 22.22 \\
\hline 39.6 & 21.36 & 21.42 & 21.41 & 21.63 & 22.36 & 4 & 22.23 \\
\hline 40.0 & 21.51 & 21.57 & & & & & 22.30 \\
\hline 40.4 & 21.69 & 22.03 & & 22 & 22 & 22 & 22.39 \\
\hline 40.8 & 21.89 & 22.50 & 22.05 & 22.65 & 23 & 22 & 22.59 \\
\hline 41.2 & 22.36 & 23.16 & 22.74 & & 23.00 & & 22.82 \\
\hline 41.6 & 23.26 & 23.22 & 23.44 & 23.52 & 22.94 & 22.83 & 23.12 \\
\hline 42.0 & 23.73 & 23.86 & 23.76 & 23.52 & 23.00 & 22.94 & 23.38 \\
\hline 42.4 & 23.98 & 24.11 & 23.68 & 23.26 & 22.89 & 22.95 & 23.62 \\
\hline 42.8 & 23.79 & 23.68 & 23.87 & 23.22 & 22.64 & 22.84 & 23.72 \\
\hline 43.2 & 23.44 & 23.24 & 23.15 & 22.79 & 22.15 & 22.57 & 23.76 \\
\hline 43.6 & 22.58 & 22.51 & 22.01 & 21.78 & 21.30 & 22.42 & 23.56 \\
\hline 44.0 & 20.88 & 20.75 & 20.49 & 20.19 & 20.62 & 22.19 & 23.48 \\
\hline 44.4 & 18.09 & 18.48 & 18.54 & 18.72 & 20.25 & 21.92 & 23.55 \\
\hline 4 & 17.34 & 17.09 & 17.14 & 18.13 & 19.80 & 21.47 & 22.88 \\
\hline 45.2 & 16.60 & 17.25 & 17.22 & 17.89 & 19.64 & 21.10 & 22.21 \\
\hline 45.6 & 16.79 & 17.30 & 17.16 & 17.85 & 19.58 & 21.26 & 22.39 \\
\hline 46.0 & 16.97 & 17.62 & 17.57 & 18.35 & 20.40 & 21.77 & 22.56 \\
\hline 46.4 & 19.69 & 19.72 & 19.79 & 20.10 & 21.37 & 22.22 & 22.74 \\
\hline
\end{tabular}


TABLE II. (Continued.)

\begin{tabular}{|c|c|c|c|c|c|c|c|}
\hline$\varepsilon$ & $300 \mathrm{~K}$ & $350 \mathrm{~K}$ & $420 \mathrm{~K}$ & $600 \mathrm{~K}$ & $900 \mathrm{~K}$ & $1200 \mathrm{~K}$ & $1675 \mathrm{~K}$ \\
\hline 46.8 & 21.63 & 21.68 & 21.43 & 21.26 & 21.85 & 22.24 & 22.91 \\
\hline 47.2 & 22.77 & 22.75 & 22.76 & 22.05 & 22.33 & 22.22 & 22.12 \\
\hline 47.6 & 22.70 & 22.44 & 22.52 & 22.42 & 22.18 & 22.02 & 21.89 \\
\hline 48.0 & 22.22 & 22.09 & 21.85 & 21.97 & 21.76 & 21.78 & 21.84 \\
\hline 48.4 & 21.42 & 21.29 & 21.52 & 21.34 & 21.36 & 21.67 & 21.90 \\
\hline 48.8 & 20.77 & 20.71 & 20.92 & 20.90 & 21.31 & 21.75 & 22.06 \\
\hline 49.2 & 20.54 & 20.37 & 20.51 & 20.93 & 21.70 & 22.28 & 22.49 \\
\hline 49.6 & 20.56 & 20.85 & 20.68 & 21.25 & 21.78 & 22.50 & 22.95 \\
\hline 50.0 & 20.82 & 20.93 & 21.05 & 21.82 & 22.18 & 22.91 & 23.43 \\
\hline 50.4 & 21.41 & 21.55 & 22.12 & 22.47 & 22.78 & 23.80 & 24.56 \\
\hline 50.8 & 22.10 & 22.73 & 22.30 & 23.16 & 23.60 & 24.90 & 25.87 \\
\hline 51.2 & 22.78 & 22.82 & 23.24 & 23.88 & 24.54 & 26.10 & 27.30 \\
\hline 51.6 & 23.46 & 23.60 & 24.13 & 24.65 & 25.57 & 27.66 & 29.44 \\
\hline 52.0 & 24.14 & 23.95 & 24.78 & 25.52 & 26.83 & 30.01 & 33.15 \\
\hline 52.4 & 24.82 & 24.95 & 25.69 & 26.71 & 28.64 & 33.19 & 37.05 \\
\hline 52.8 & 25.50 & 26.22 & 26.59 & 28.55 & 31.27 & 36.80 & 41.10 \\
\hline 53.2 & 26.92 & 27.92 & 28.57 & 31.45 & 34.78 & 40.64 & 42.90 \\
\hline 53.6 & 28.81 & 30.39 & 31.91 & 35.79 & 39.12 & 43.55 & 44.21 \\
\hline 54.0 & 33.72 & 35.25 & 36.46 & 40.81 & 43.47 & 45.28 & 45.17 \\
\hline 54.4 & 39.48 & 41.58 & 42.07 & 45.27 & 46.22 & 46.54 & 44.84 \\
\hline 54.8 & 46.78 & 46.45 & 46.97 & 48.32 & 47.80 & 46.23 & 42.15 \\
\hline 55.2 & 51.72 & 51.76 & 50.88 & 50.07 & 48.52 & 43.32 & 39.14 \\
\hline 55.6 & 55.17 & 53.09 & 52.15 & 49.87 & 46.22 & 39.19 & 35.92 \\
\hline 56.0 & 53.24 & 51.53 & 50.47 & 46.70 & 41.48 & 34.11 & 30.03 \\
\hline 56.4 & 49.78 & 47.69 & 45.99 & 40.70 & 34.72 & 27.12 & 23.69 \\
\hline 56.8 & 41.38 & 39.09 & 37.19 & 32.72 & 26.53 & 20.00 & 16.98 \\
\hline 57.2 & 31.52 & 30.01 & 28.03 & 23.60 & 18.15 & 13.48 & 11.51 \\
\hline 57.6 & 19.42 & 18.90 & 17.58 & 14.95 & 11.01 & 8.80 & 7.93 \\
\hline 58.0 & 10.37 & 9.81 & 9.52 & 7.99 & 5.88 & 5.59 & 4.64 \\
\hline 58.4 & 2.90 & 3.51 & 3.56 & 3.48 & 3.32 & 2.90 & 1.50 \\
\hline 58.8 & 1.08 & 1.24 & 1.27 & 1.63 & 1.47 & 1.06 & 0.76 \\
\hline 59.2 & 0.00 & 0.20 & 0.38 & 0.66 & 0.54 & 0.48 & 0.29 \\
\hline 59.6 & 0.00 & 0.06 & 0.11 & 0.25 & 0.27 & 0.31 & 0.13 \\
\hline 60.0 & 0.00 & 0.01 & 0.06 & 0.11 & 0.19 & 0.18 & 0.23 \\
\hline 60.4 & 0.00 & 0.01 & 0.02 & 0.07 & 0.15 & 0.24 & 0.66 \\
\hline 60.8 & 0.00 & 0.02 & 0.03 & 0.06 & 0.16 & 0.53 & 1.48 \\
\hline 61.2 & 0.00 & 0.01 & 0.02 & 0.05 & 0.27 & 1.13 & 2.46 \\
\hline 61.6 & 0.00 & 0.01 & 0.04 & 0.08 & 0.53 & 1.88 & 4.04 \\
\hline 62.0 & 0.00 & 0.03 & 0.07 & 0.14 & 0.94 & 3.10 & 5.57 \\
\hline 62.4 & 0.00 & 0.05 & 0.10 & 0.28 & 1.61 & 4.46 & 6.86 \\
\hline 62.8 & 0.08 & 0.16 & 0.28 & 0.57 & 2.60 & 5.71 & 8.22 \\
\hline 63.2 & 0.26 & 0.44 & 0.66 & 1.12 & 3.73 & 6.88 & 9.64 \\
\hline
\end{tabular}

TABLE II. (Continued.)

\begin{tabular}{|c|c|c|c|c|c|c|c|}
\hline$\varepsilon$ & $300 \mathrm{~K}$ & $350 \mathrm{~K}$ & $420 \mathrm{~K}$ & $600 \mathrm{~K}$ & $900 \mathrm{~K}$ & $1200 \mathrm{~K}$ & $1675 \mathrm{~K}$ \\
\hline 3.6 & 0.82 & 0.98 & 8 & 1.91 & 3 & 0 & .92 \\
\hline 4.0 & 1.51 & 1.74 & & 2.89 & & .60 & 49 \\
\hline 64.4 & 2.50 & 2.80 & 3.04 & 3.85 & 6.86 & 11.60 & 17.32 \\
\hline 64.8 & 3.45 & 3.74 & 3.99 & 4.73 & 8.05 & 13.94 & 19.75 \\
\hline 5.2 & 4.25 & 4.51 & 4.91 & 5.60 & 9.49 & 16.83 & 21.05 \\
\hline 5.6 & 5.06 & 5.40 & 5.67 & 6.55 & 11.28 & & 21.48 \\
\hline 66.0 & 5.85 & 6.29 & 6.54 & 7.66 & 13.44 & 22.21 & 21.12 \\
\hline 5.4 & 6.90 & 7.20 & 7.70 & 8.97 & 16.69 & & 19.69 \\
\hline 66.8 & 8.02 & 8.67 & 9.11 & 10.54 & 21.15 & 23.09 & 17.93 \\
\hline 67.2 & 9.41 & 9.84 & 10.69 & 12.50 & 24.13 & 21.64 & 15.56 \\
\hline 67.6 & 11.02 & 12.11 & 13.48 & 16.19 & 25 & 19 & 13.39 \\
\hline 68.0 & 13.09 & 14.71 & 16.95 & 21.42 & 25.67 & 16 & 11.63 \\
\hline 3.4 & 17.55 & 19.41 & 21.15 & 25.68 & 24.04 & & 10.23 \\
\hline 68.8 & 23.78 & 24.93 & 25.56 & 28.02 & 21.09 & 37 & 9.09 \\
\hline 69.2 & 29.06 & 28.36 & 28.91 & 28.64 & 17.76 & & 8.20 \\
\hline 69.6 & 33.36 & 31.32 & 29.93 & 26.61 & 15.07 & & 7.37 \\
\hline 70.0 & 30.18 & 28.5 & 27.3 & 22.78 & 12. & & 6.74 \\
\hline 70.4 & 26.29 & 24.18 & 23.46 & 18.82 & 11 & 7.75 & 6.07 \\
\hline 70.8 & 21.14 & 20.50 & 18.61 & 15.75 & & & 5.33 \\
\hline 71.2 & 17.38 & 16.86 & 15.90 & 13.39 & 6 & 3 & 4.89 \\
\hline 71.6 & 14.49 & 14.11 & 13.2 & 11.62 & 7.9 & & 4.66 \\
\hline 7 & 12. & 11. & & & & & 4.42 \\
\hline 72.4 & 10.81 & 10.40 & 10 & 9.05 & & & 4.19 \\
\hline 72.8 & 9.58 & & & 8.08 & & 4. & 4.03 \\
\hline 73.2 & 8.51 & 2 & 7.8 & 7.29 & 5 & 4.46 & 3.82 \\
\hline 73.6 & 7.6 & 7.3 & 7.2 & 6.67 & & 0 & 3.54 \\
\hline 74.0 & 6.97 & 6.70 & 64 & 6.15 & 4. & 3.92 & 3.29 \\
\hline 74.4 & 6.3 & 6.22 & & & & & 3.06 \\
\hline 74.8 & & & & & & & 2.98 \\
\hline 75.2 & 5.4 & & & & & & 2.93 \\
\hline 75.6 & 5.1 & 5.06 & 5. & & & 3. & 2.92 \\
\hline 76.0 & 4.8 & 4.8 & & 4.43 & 3. & 3. & 2.67 \\
\hline 76.4 & 4.59 & & & & & 2.79 & 2.12 \\
\hline 76.8 & 4.42 & 4.31 & 4.40 & 4.05 & 3.22 & 2.19 & 1.33 \\
\hline 77.2 & 4.29 & 4.11 & 3.94 & 3.78 & 2.71 & 1.47 & 0.44 \\
\hline 77.6 & 4.05 & 3.81 & 3.94 & 3.45 & & 1.00 & 0.18 \\
\hline 78.0 & 3.74 & 3.61 & 3.68 & 3.10 & 1.88 & 0.75 & 0.00 \\
\hline 78.4 & 3.16 & 2.83 & 2.96 & 2.63 & 1.56 & 0.61 & 0.00 \\
\hline 78.8 & 2.47 & 2.33 & 2.28 & 2.13 & 1.26 & 0.49 & 0.00 \\
\hline 79. & 1.6 & 1.62 & 1.86 & 1.92 & 1.17 & 0.48 & 0.00 \\
\hline 79.6 & 0.98 & 1.20 & 1.29 & 1.92 & 1.17 & 0.48 & 0.00 \\
\hline 80.0 & 0.00 & 0.00 & 0.00 & 0.00 & 0.00 & 0.00 & 0.00 \\
\hline
\end{tabular}

[1] I. M. Thorson and B. C. Haywood, Scattering law for $\mathrm{UO}_{2}$, in Proceedings of a Symposium on Inelastic Scattering of Neutrons in Solids and Liquids, IAEA Rep. No. STI/PUB/62 (IAEA, Vienna, 1963), Vol. 2, p. 213.

[2] Purohit et al., Inelastic neutron scattering in metal hydrides, UC and $\mathrm{UO}_{2}$, and applications of the scattering law, in Proceedings of a Symposium on Neutron Thermalization and Reactor Spectra, IAEA Rep. No. STI/PUB/160 (IAEA, Vienna, 1967), Vol. 1, p. 407.

[3] D. Brown et al., Nucl. Data Sheets 148, 1 (2018).
[4] A. I. Hawari et al., Nucl. Data Sheets 118, 172 (2014).

[5] G. Kresse and J. Furthmuller, Comput. Matter. Sci. 6, 15 (1996).

[6] J. L. Wormald and A. I. Hawari, Am. Nucl. Soc. 115, 1156 (2016).

[7] G. Dolling, R. A. Cowley, and A. D. Woods, Can. J. Phys. 43, 1397 (1965).

[8] G. Noguere, P. Maldonado, and C. de Saint Jean, Eur. Phys. J. Plus 133, 177 (2018).

[9] Y. Yun, D. Legut, and P. M. Oppeneer, J. Nucl. Mater. 426, 109 (2012). 
[10] P. Maldonado, L. Paolasini, P. M. Oppeneer, T. R. Forrest, A. Prodi, N. Magnani, A. Bosak, G. H. Lander, and R. Caciuffo, Phys. Rev. B 93, 144301 (2016).

[11] A. Togo, https://atztogo.github.io/phonopy/.

[12] J. W. L. Pang, W. J. L. Buyers, A. Chernatynskiy, M. D. Lumsden, B. C. Larson, and S. R. Phillpot, Phys. Rev. Lett. 110, 157401 (2013).

[13] J. W. L. Pang, A. Chernatynskiy, B. C. Larson, W. J. L. Buyers, D. L. Abernathy, K. J. McClellan, and S. R. Phillpot, Phys. Rev. B 89, 115132 (2014).

[14] M. S. Bryan, J. W. L. Pang, B. C. Larson, A. Chernatynskiy, D. L. Abernathy, K. Gofryk, and M. E. Manley, Phys. Rev. Mater. 3, 065405 (2019).

[15] B. Becker et al., Ann. Nucl. Ener. 36, 281 (2009).

[16] E. Brun et al., Ann. Nucl. Ener. 82, 151 (2015).

[17] R. E. MacFarlane, The NJOY nuclear data processing system, Version 2016, Los Alamos National Laboratory Rep. No. LAUR-17-20093 (Los Alamos National Laboratory, Los Alamos, 2019).

[18] L. Van Hove, Phys. Rev. 95, 249 (1954).

[19] A. W. Solbrig, Am. J. Phys. 29, 257 (1961).

[20] A. Sjolander, Arkiv Fysik 14, 315 (1958).

[21] S. Xu et al., Measurement of the double-differential neutron cross section of $\mathrm{UO}_{2}$ from room temperature to hot full power conditions, in Proceedings of the 11th International Conference on Nuclear Criticality Safety, Paris, France, 2019 (American Nuclear Society, La Grange Park, 2019).

[22] G. Noguere et al., EPJ Web Conf. 239, 14003 (2002).

[23] J. Dawidowski et al., Nucl. Instrum. Meth. B 195, 389 (2002).

[24] A. A. Maradudin and A. E. Fein, Phys. Rev. 128, 2589 (1962).

[25] J. J. J. Kokkedee, Physica 28, 374 (1962).

[26] P. Archier et al., Nucl. Data Sheets 118, 488 (2014).

[27] T. Feng, X. Yang, and X. Ruan, J. Appl. Phys. 124, 145101 (2018).

[28] M. T. Hutchings, J. Chem. Soc., Faraday Trans. 83, 1083 (1987).

[29] A. S. Poplavnoi and T. P. Fedorova, Moscow Univ. Phys. Bull. 65, 397 (2010).

[30] B. T. M. Willis, J. Phys. France 25, 431 (1964).

[31] L. Desgranges et al., Inorg. Chem. 56, 321 (2017).

[32] Y. Ma et al., Inorg. Chem. 57, 7064 (2017).

[33] H. Serizawa et al., J. Alloys Compd. 271-273, 386 (1998).

[34] P. Ruello et al., J. Phys. Chem. Solids 66, 823 (2005).

[35] B. T. M. Willis, Proc. R. Soc. London A 274, 122 (1963).

[36] B. T. M. Willis, Proc. R. Soc. London A 274, 134 (1963).

[37] A. Albinati, Acta Cryst. A 36, 265 (1980). 